\title{
CHARACTERIZATION OF ACTIVE GALACTIC NUCLEI AND THEIR HOSTS IN THE EXTENDED GROTH STRIP: A MULTIWAVELENGTH ANALYSIS
}

\author{
C. Ramos Almeida ${ }^{1}$, J. M. Rodríguez Espinosa ${ }^{1}$, G. Barro ${ }^{2}$, J. Gallego ${ }^{2}$, and P. G. Pérez-GonzÁlez ${ }^{2,3}$ \\ ${ }^{1}$ Instituto de Astrofísica de Canarias (IAC), C/Vía Láctea, s/n, E-38205, La Laguna, Tenerife, Spain; cra@iac.es, jre@iac.es \\ ${ }^{2}$ Departamento de Astrofísica y Ciencias de la Atmósfera, Facultad de Ciencias Físicas, Universidad Complutense de Madrid, E-28040 Madrid, Spain; \\ gbc@astrax.fis.ucm.es, jgm@astrax.fis.ucm.es, pgperez@astrax.fis.ucm.es \\ ${ }^{3}$ Associate Astronomer at Steward Observatory, University of Arizona, Tucson, AZ 85721, USA \\ Received 2008 June 25; accepted 2008 October 7; published 2008 December 10
}

\begin{abstract}
We have employed a reliable technique of classification of active galactic nuclei (AGNs) based on the fit of well sampled spectral energy distributions (SEDs) with a complete set of AGN and starburst galaxy templates. We have compiled UV, optical, and IR data for a sample of 116 AGNs originally selected for their X-ray and mid-IR emissions ( 96 with single detections and 20 with double optical counterparts). This is the most complete compilation of multiwavelength data for such a large sample of AGN in the Extended Groth Strip. Through these SEDs, we are able to obtain highly reliable photometric redshifts and to distinguish between pure and host-dominated AGNs. For the objects with unique detection we find that they can be separated into five main groups, namely: Starburst-dominated AGNs (24\% of the sample), Starburst-contaminated AGNs (7\%), Type-1 AGNs (21\%), Type-2 AGNs (24\%), and Normal galaxy hosting AGN (24\%). We find these groups concentrated at different redshifts: Type-2 AGNs and Normal galaxy hosting AGNs are concentrated at low redshifts, whereas Starburst-dominated AGNs and Type-1 AGNs show a larger span. Correlations between hard/soft X-ray and UV, optical and IR luminosities are reported for the first time for such a sample of AGNs spanning a wide range of redshifts. For the 20 objects with double detection, the percentage of Starburst-dominated AGNs increases up to $48 \%$.
\end{abstract}

Key words: galaxies: active - galaxies: nuclei - galaxies: starburst - infrared: galaxies - ultraviolet: galaxies X-rays: galaxies

Online-only material: color figures

\section{INTRODUCTION}

The role of active galactic nuclei (AGNs) in the formation and evolution of galaxies is still not well established. It is not clear whether AGNs represent episodic phenomena in the life of galaxies, are random processes (given that the supermassive black hole is already there), or are more fundamental. Some authors claim that AGNs are key in quenching the star-formation bursts in their host galaxies (Granato et al. 2004; Springel et al. 2005). It has also been shown that the mass dependence of the peak star formation epoch appears to mirror the mass dependence of black hole (BH) activity, as recently seen in redshift surveys of both radio- and X-ray-selected AGNs (Waddington et al. 2001; Hasinger 2004). For these reasons, searching for signatures of AGN feedback in the properties of AGN host galaxies is one of the most promising ways of testing the role of AGNs in galaxy evolution.

One way of finding variations in the AGNs population with redshift is to compare their spectral energy distributions (SEDs) defined over a broad wavelength range. The SED of an AGN can reveal the presence of the underlying central engine, together with the luminosity of the host galaxy, the reddening, and the role of the star formation in the various frequency regimes. SED determination in samples of AGNs at different redshifts is an efficient method to search for evolutionary trends. Accuracy in the photometry and a filter set spanning a broad wavelength range are required to correctly characterize different types of AGNs.

Multiwavelength surveys are fundamental in the study of AGNs, since these appear considerably different depending on the wavelength range of consideration. The hard X-ray selection of AGN using deep observations is one of the most reliable methods of finding AGNs (Mushotzky 2004), although a percentage of them remains undetected using this technique (Peterson et al. 2006), especially the most highly obscured objects. For this reason, it is important to characterize AGNs at different wavelength ranges, in order to be capable of identifying them by more than one selection technique, and to distinguish between the different groups of active nuclei, including those that could be contaminated, or even hidden, by starbursts. Mid-IR surveys have been very successful in finding X-ray undetected AGNs in large numbers, but in this case it is crucial to distinguish the AGNs from the nonactive star-forming galaxies. This can be achieved using typical mid-IR colors of AGNs (Lacy et al. 2004; Stern et al. 2005; Alonso-Herrero et al. 2006; Donley et al. 2008) or by combining mid-IR and radio detections (Donley et al. 2005; Alonso-Herrero et al. 2006; MartínezSansigre et al. 2005, 2007; Lacy et al. 2007; Park et al. 2008).

The Extended Groth Strip (EGS; $\alpha=14^{\mathrm{h}} 17^{\mathrm{m}}, \delta=52^{\circ} 30^{\prime}$ ) enlarges the Hubble Space Telescope Groth-Westphal strip (Groth et al. 1994) up to $2^{\circ} \times 15^{\prime}$, having the advantage of being a low extinction area in the northern sky, with low galactic and zodiacal IR emission, and good schedulability by space observatories. For these reasons, there is a huge amount of public data at different wavelength ranges that only need to be compiled and cross-correlated in a consistent way. The overall majority of the observational work in the EGS have been coordinated by the AEGIS project ${ }^{4}$ (Davis et al. 2007).

\footnotetext{
4 The AEGIS project is a collaborative effort to obtain both deep imaging covering all major wavebands from X-ray to radio and optical spectroscopy over a large area of sky (http://aegis.ucolick.org/index.html).
} 
With the huge amount of data available for this region of the sky, we have constructed a robust AGN sample, detected in the X-rays and in the mid-IR, intermediate in depth and area in comparison with other surveys (Jannuzi \& Dey 1999; Dickinson et al. 2001; Lonsdale et al. 2003; Eisenhardt et al. 2004; Franceschini et al. 2005). The photometry has been performed over the publicly available images, in several bands, in order to compile as best-sampled SEDs as possible. The biggest advantage of our AGN sample, compared with other works, is the robustness of the photometry, performed in a consistent way among the different bands, and its multiwavelength nature: it is the most complete compilation of data for such a big sample of AGNs in the EGS. This allows us to determine accurate photometric redshifts, and to clearly distinguish between the different groups of AGNs. We have used a comprehensive set of AGNs plus starburst templates from Polletta et al. (2007) to fit the SEDs of the galaxies in the sample, and to separate them in five different main groups. Through this classification, we can study the properties of the different types of AGNs in this sample. Section 2 describes the sample and the cross-matching of the multiwavelength data, Section 3 explains the technique of classification of AGNs based on the fit of their SEDs, in Section 4 the results of this paper are discussed, and finally in Section 5 the main conclusions are summarized. Throughout this paper we assume an $H_{0}=75 \mathrm{~km} \mathrm{~s}^{-1} \mathrm{Mpc}^{-1}$ and a $\Lambda$ cold dark matter $(\mathrm{CDM})$ cosmology with $\Omega_{m}=0.3$ and $\Omega_{\Lambda}=0.7$.

\section{SAMPLE AND MULTIWAVELENGTH DATA}

The sample studied in this work, which comprises 116 AGN candidates, was built from the previously published X-ray catalogs found in Waskett et al. (2003), Nandra et al. (2005), and Barmby et al. (2006). These objects were originally selected by Barmby et al. (2006), both in the X-ray (Chandra and $X M M-N e w t o n)$ and in the mid-IR (Spitzer). The X-ray and mid-IR observations in the EGS are intermediate in depth and area between GOODS (Dickinson et al. 2001), the shallower NOAO Deep-Wide Field (Jannuzi \& Dey 1999; Eisenhardt et al. 2004), and SWIRE (Lonsdale et al. 2003) surveys. Therefore, this region provides a valuable test of AGN properties at intermediate fluxes. In addition to this, we have compiled UV, optical, and near-IR (NIR) archival data for these AGNs, in order to increase the definition of their SEDs. These well-sampled SEDs allow us, first, to classify the objects in different galaxy population groups and calculate their photometric redshifts, and second, to study the physical properties of this representative sample of AGNs.

The Chandra data were taken with the Advanced CCD Imaging Spectrometer Imager (ACIS-I) in 2002 August (Nandra et al. 2005), consisting of a $200 \mathrm{ks}$ exposure with a limiting full-band flux $(0.5-10 \mathrm{keV})$ of $3.5 \times 10^{-16} \mathrm{erg} \mathrm{cm}^{-2} \mathrm{~s}^{-1}$. The XMM-Newton data were obtained in 2000 July with a $56 \mathrm{ks}$ exposure and with a limiting $0.5-10 \mathrm{keV}$ flux of $2 \times$ $10^{-15} \mathrm{erg} \mathrm{cm}^{-2} \mathrm{~s}^{-1}$ (Waskett et al. 2003). Barmby et al. (2006) combined both catalogs producing a list of 152 sources within the limits of the Spitzer mid-IR observations.

The Spitzer data (Infrared Array Camera (IRAC) and (Multiband Imaging Photometer for Spitzer (MIPS)) are part of the IRAC Deep Survey, taken during 2003 December and 2004 June-July with $2.7 \mathrm{hr}$ exposure per pointing. In the case of the MIPS data, the observations were done in 2004 January and June with a depth of $1200 \mathrm{~s}$ per pointing. The $5 \sigma$ limiting AB magnitudes are 24.0, 24.0, 21.9, and 22.0 for the IRAC bands, and 19.1 in the case of MIPS. Barmby et al. (2006) finally selected 138 objects with secure detections in all four IRAC bands, out of the $152 \mathrm{X}$-ray emitters. The detection of these objects in both the X-rays and the mid-IR gives confidence in their classification as AGNs. Besides, we have also checked that the values of the hard X-ray and $24 \mu \mathrm{m}$ fluxes lie inside the AGN-characteristic region (see Figure 1 of Alonso-Herrero et al. 2004).

In addition to the previous, we have compiled near- and farUV (NUV and FUV, respectively) images from the GALEX GR2/GR3 data release ( $3 \sigma$ limiting $\mathrm{AB}$ magnitudes $=25$ in both FUV and NUV filters); optical images from the CanadaFrance-Hawaii Telescope (CFHT) Legacy Survey (CHFTLS), T0003 worldwide release (S. Gwyn et al. 2009, in preparation), taken with the MegaCam imager on the $4 \mathrm{~m} \mathrm{CFHT} \mathrm{(Boulade} \mathrm{et}$ al. 2003; $5 \sigma$ limiting $\mathrm{AB}$ magnitudes $=26.3$, 27.0, 26.5, 26.0, and 25.0 in $u, g, r, i$, and $z$ bands); and $J$ and $K_{S}$ data from the version 3.3 of the Palomar-WIRC $K$-selected catalog of Bundy et al. (2006; $5 \sigma$ limiting Vega magnitudes $=23$ and 20.6 in the $J$ and $K_{S}$ bands).

The fluxes employed in this work have been measured in a compilation of publicly available imaging data, which is outlined briefly in Villar et al. (2008) and will be described in detail in G. Barro et al. 2009, in preparation (see also PérezGonzález et al. 2008b). Photometry in consistent apertures was measured in all bands with available imaging data following the procedure described in Pérez-González et al. (2005, 2008a). In the NIR, no deep $J$ - and $K_{S}$-band imaging data were available and we have used the photometric catalogs published by Bundy et al. (2006). The same happens with the X-ray data that have been drawn from the catalogs (Waskett et al. 2003; Nandra et al. 2005; Barmby et al. 2006).

We have performed the cross-matching of the 138 sources between the X-ray and Spitzer data, adding UV, optical, and NIR data points, avoiding the false matches that Barmby et al. (2006) expected in their sample. We identify these objects through their IRAC positions in our merged photometric catalog (Pérez-González et al. 2005, 2008a). The source coordinates on the IRAC $3.6 \mu \mathrm{m}$ images are then cross-correlated with each one of the UV, optical, and NIR catalogs using a search radius of 2 ".5, starting with the deepest images. When a source is identified in one of these images, the Kron (1980) elliptical aperture from this reference image is taken and overlaid onto each of the other bands. The aperture employed is large enough to enclose the point-spread function (PSF) in all the UV, optical, and NIR images (the seeing being less than 1".5). For IRAC and MIPS, because of their large PSFs, integrated magnitudes measured in small apertures (applying aperture corrections) are employed. The hard and soft X-ray fluxes are obtained by cross-correlating the IRAC positions with the X-ray catalogs, using a search radius of $2^{\prime \prime}$ in this case. Uncertainties of each measured flux are obtained from the sky pixel-to-pixel variations, detector readout noise, Poisson noise in the measured fluxes, errors in the World Coordinate System, and errors in the absolute photometric calibration.

In some cases, for a single IRAC source, there are several counterparts in the ground-based images within the 2 ".5 search radius. For these objects, the ground-based optical/NIR reference image is used to determine the positions of each source separately. The IRAC images are then deconvolved using the IRAC PSFs. Although the IRAC PSFs have FWHMs of approximately $2^{\prime \prime}$, the determination of the central position of each IRAC source can be performed more accurately, and sources can be resolved for separations $\sim 1^{\prime \prime}$ from each other. IRAC 
fluxes are then remeasured by fixing the positions of the objects in each pair, and by scaling the flux of each object in an aperture of 0.9 . For a more detailed description of the cross-matching and aperture photometry see Pérez-González et al. (2005, 2008a).

Out of the 138 sources that comprise the final sample chosen by Barmby et al. (2006), we find 96 sources that have unique detections in all bands, plus another 20 objects with double detection in the ground-based images. We discard the remaining 22 objects because 21 of them show multiple (more than two) detections in the optical/NIR images, leading to possible source confusion, and the other object shows a starlike SED. The analysis of the data will be done first for the 96 objects that are definitely free from contamination from other sources. Nevertheless, in Section 4.5, we analyze the images and photometric redshifts of those additional 20 objects with double detection.

\section{SPECTRAL ENERGY DISTRIBUTIONS AND PHOTOMETRIC REDSHIFTS OF OBJECTS WITH UNIQUE DETECTION}

In order to classify the 96 SEDs and to estimate their photometric redshifts, we combine optical $(u, g, r, i, z)$, NIR $(J, K)$, and mid-IR data (IRAC 3.6, 4.5, 5.8, $8 \mu \mathrm{m}$ and MIPS $24 \mu \mathrm{m}$ ) to build well-sampled SEDs that we then fit with the library of starburst, AGN, and galaxy templates taken from Polletta et al. (2007). We make use of the photometric redshift code HyperZ (Bolzonella et al. 2000) to perform the fits. This code determines the best photometric redshifts $\left(z_{\text {phot }}\right)$ by minimization of the $\chi^{2}$ derived from a comparison between the photometric SEDs and the set of template spectra, leaving the redshift as a variable. The code also takes into account the effects of dust extinction according to the selected reddening law (Calzetti et al. 2000). Choosing a wide range of reddening values seems to be essential to reproduce the SEDs of high redshift galaxies (Bolzonella et al. 2000). According to Steidel et al. (1999), the typical $E(B-V)$ for galaxies up to $z \sim 4$ is $0.15 \mathrm{mag}$, thus $A_{V} \sim 0.6$ mag when using Calzetti's law. The maximum $A_{V}$ allowed in our calculations is about 2 times this value, thus $A_{V}$ ranges from 0.0 to 1.2 , with a step between them of 0.3 . Similar values of $A_{V}$ are typically chosen in the literature (Bolzonella et al. 2000; Babbedge et al. 2004).

The chosen set of templates contains 23 SED types that we have arranged into the following five main groups: Starburst-dominated AGNs (which includes four Starbursts and Starburst/ULIRGs (ultraluminous IR galaxies) templates), Starburst-contaminated AGNs (three templates, namely, Starburst/ULIRG/Seyfert 1, Starburst/Seyfert 2, and Starburst/ ULIRG/Seyfert 2), Type-1 AGNs (three Type-1 QSO templates), Type-2 AGNs (Type-2 QSO, Torus-QSO, Seyfert 1.8, and Seyfert 2 templates), and finally, Normal galaxy hosting AGNs (nine templates including 2, 5, and $13 \mathrm{Gyr}$ ellipticals plus $\mathrm{S} 0, \mathrm{Sa}, \mathrm{Sb}, \mathrm{Sc}, \mathrm{Sd}$, Sdm-type spirals). These templates span the range in wavelength between 0.1 and $1000 \mu \mathrm{m}$. For a detailed explanation of their synthesis see Polletta et al. (2007). Our main interest is to classify all of our sources into these five main groups and to determine the distribution of the sources into each of these groups. Note that although all of the sources are AGNs, the Starburst-dominated AGNs have their SEDs dominated by the starburst emission from the optical to the mid-IR; the Normal galaxy hosting AGNs would be low-luminosity AGNs embedded in an otherwise normal galaxy emission; and in the case of the Starburst-contaminated
AGNs, the emission of both the starburst or the AGN dominate depending on which wavelength is being looked at. Indeed, some of these objects noticeably show the AGN power law beyond the NIR.

We fit data from the optical $u$ band up to the MIPS $24 \mu \mathrm{m}$ band. We avoid the use of GALEX data because few galaxies have these, and because their use introduces large errors in the fits. As explained in Polletta et al. (2007), including mid-IR data considerably improves the photometric redshift calculations, since some spectral types suffer degeneration that is broken by the nonextinguished longer wavelengths, even if the errors in the magnitudes are larger in the mid-IR than in the optical and NIR bands.

Examples of HyperZ fits for each of the employed templates are shown in Figure 1. In the Type-2 AGNs panel, only three templates are shown because none of the 96 galaxies were fitted with the Torus-QSO template. The Normal galaxy hosting AGNs panel contains only one example of elliptical template (the 2 Gyr elliptical) and one example of spiral (Sb). See Table 1 to check the SED types and their corresponding group. Photometric redshifts derived from the fits are reported in Table 1, together with the $\chi_{v}^{2}$ and probabilities given by HyperZ, the $A_{V}$, and the template used for the fit of each galaxy. In the cases where spectroscopic redshifts are available, these are also given in Table 1.

A comparison between the photometric and spectroscopic redshifts for the 39 sources with publicly available $z_{\text {spec }}$ from the Deep Extragalactic Evolutionary Probe (DEEP) data archive (Weiner et al. 2005) is shown in Figure 2. Note that only 31 out of these 39 galaxies have reliable spectroscopic redshifts (flag $=3$ or 4 in the DEEP data archive). Horizontal error bars indicating the reliability of the $z_{\text {spec }}$ are represented in Figure 2, together with vertical error bars that indicate the discrepancies between the $z_{\text {spec }}$ and $z_{\text {phot }}$. The dashed lines represent $20 \%$ agreement in $(1+z)$. The fractional error $\Delta z=\left(z_{\text {phot }}-z_{\text {spec }}\right) /\left(1+z_{\text {spec }}\right)$ quantifies the number of catastrophic outliers, which are those with $|\Delta z|>0.2$. Our measured mean $\Delta z$ for the 39 sources with spectroscopic redshifts is 0.05 , with a $\sigma_{z}=0.37$, and an outlier fraction of $\sim 18 \%$, corresponding to seven discordant objects, labeled in Figure 2. However, if we consider only the 31 objects with reliable $z_{\text {spec }}$ (flags $=$ 3 or 4$), \Delta z=-0.03$, and $\sigma_{z}=0.11$, with three outliers $(8 \%)$. These results point to the goodness of our fits, and thus we rather trust our photometric redshifts better than the spectroscopic ones for the outliers indicated (all of them with $z_{\text {spec }}$ with flags $=1$ or 2 in the DEEP database). We nevertheless note a slight underestimation of our photometric redshifts (see Figure 2) in comparison with the spectroscopic ones $(\Delta z=$ $-0.03)$. Although this effect is negligible, we are aware of it, and we assume that all the calculated $z_{\text {phot }}$ values might be affected by this slight underestimation.

Based on the good agreement between spectroscopic and photometric redshifts in this fairly large subsample of sources (the results shown are better than those typically obtained for AGN samples (Babbedge et al. 2004; Kitsionas et al. 2005; Bundy et al. 2008) and with practically the same $\sigma_{z}$ and outlier fraction than those reported by Polletta et al. 2007), we can confidently extrapolate the results to the rest of the sample. This, together with the SED classification into the five groups described above, allows us to perform a reliable statistical analysis of the different AGN populations.

It is worth mentioning that the distribution of object type in the subsample with spectroscopic redshifts is completely different 


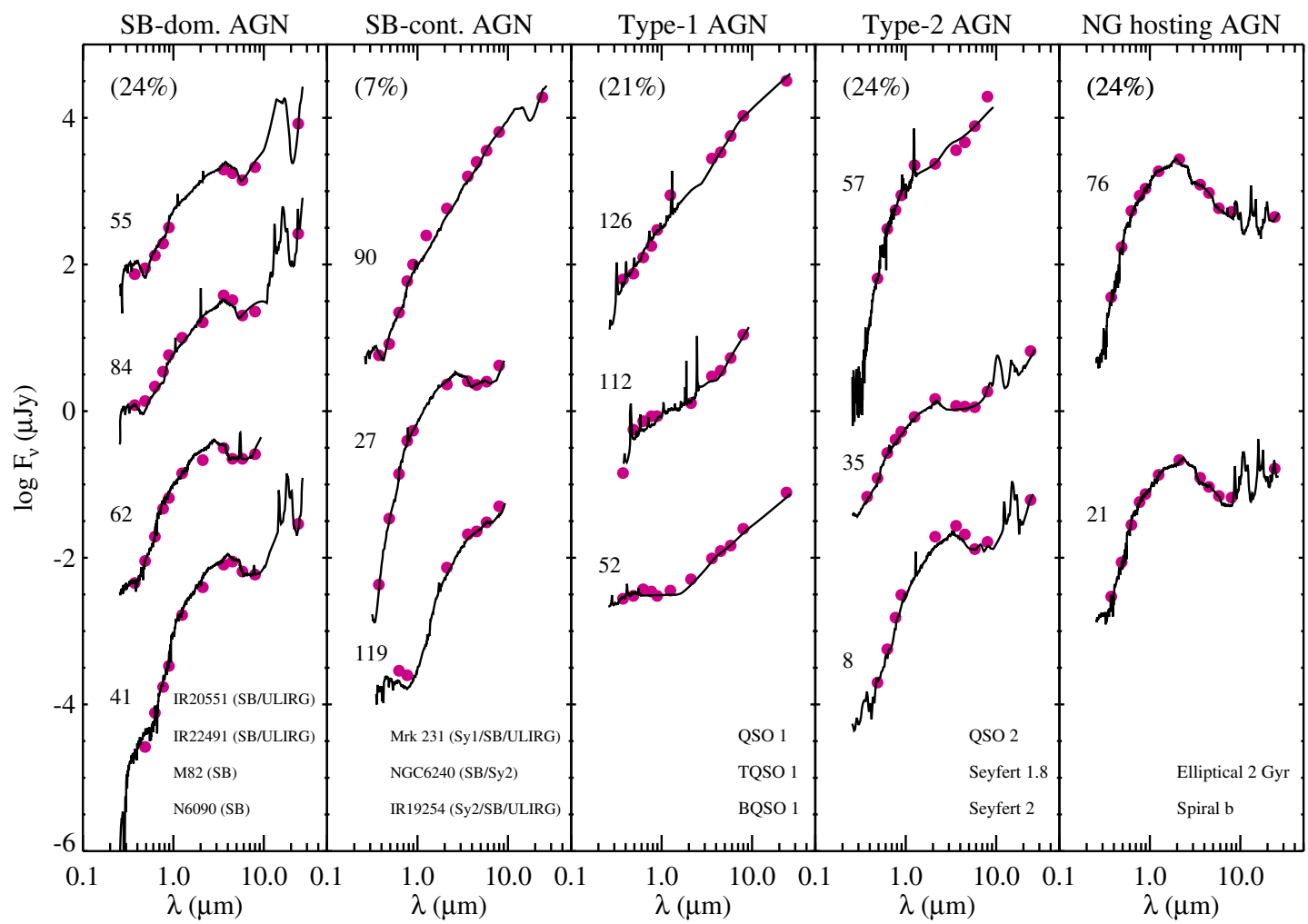

Figure 1. Examples of SEDs in our sample (dots) fitted with different templates from Polletta et al. (2007) for the five main groups considered. The legends in the bottom right of each panel refer to the actual template within the groups from Polletta et al. (2007). The data have been scaled for clarity. The $x$-axis corresponds to observed wavelength. The 15 galaxies represented here are labeled with the ID from Table 1 . The percentages of objects enclosed in each group are typed in the upper left corner of each panel.

(A color version of this figure is available in the online journal.)

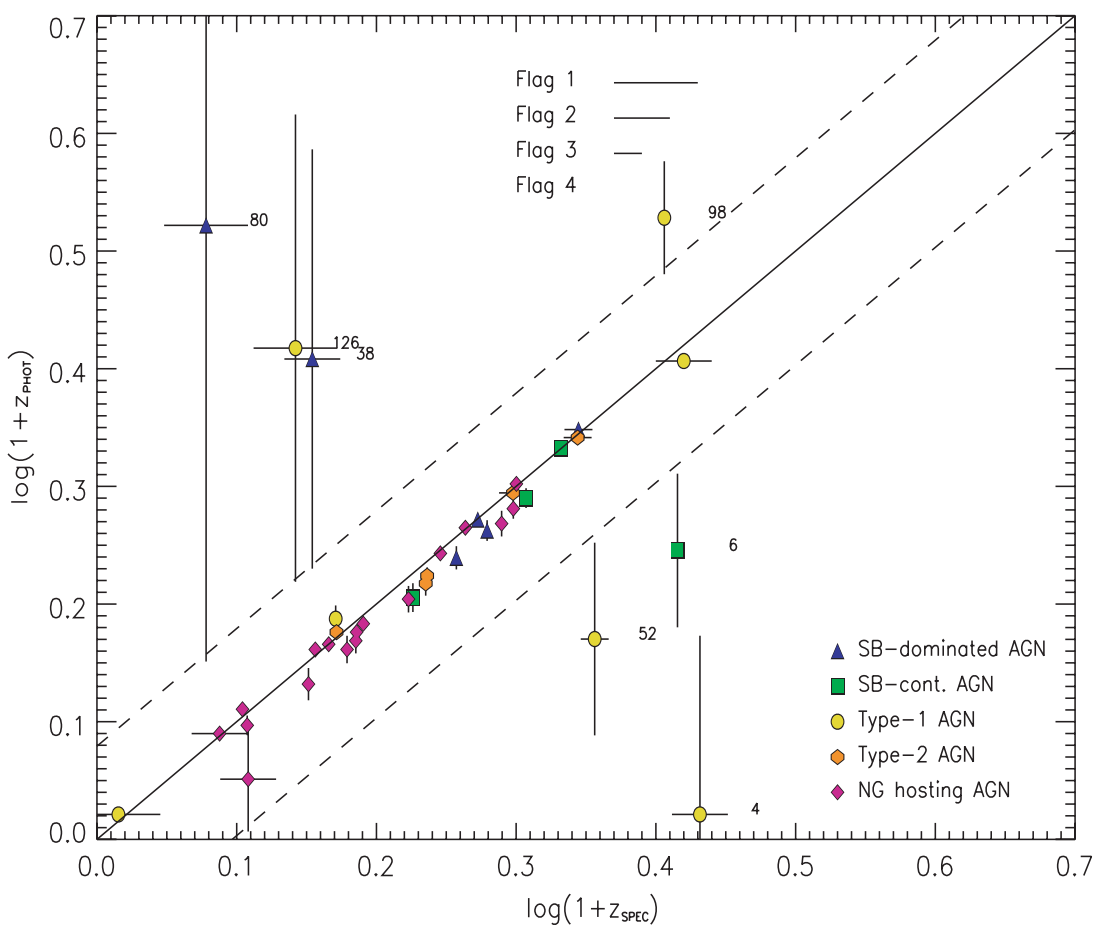

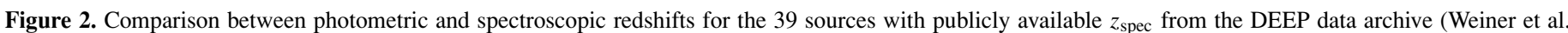

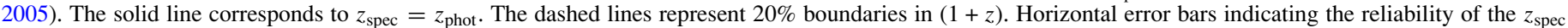

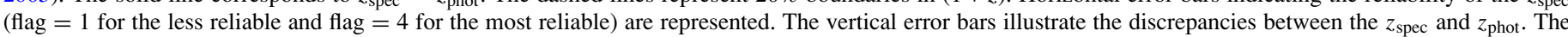
seven outliers are labeled $\left(4,6,38,52,80,98\right.$, and 126). Note that there are only three outliers when only the 31 galaxies with reliable $z_{\text {spec }}$ are considered.

(A color version of this figure is available in the online journal.) 
Table 1

Photometric Redshifts and Additional Information Derived from the SED Fits of the 96 Objects with Single Detections. Spectroscopic Redshifts are Reported When Available

\begin{tabular}{|c|c|c|c|c|c|c|c|c|c|c|c|}
\hline ID & ID IRAC & R.A. $\left({ }^{\circ}\right)$ & Decl. $\left({ }^{\circ}\right)$ & $z_{\text {spec }}$ & $z_{\text {phot }}$ & $\chi_{v}^{2}$ & Prob (\%) & $A_{V}$ & $L_{r}$ & Template & Group \\
\hline 1 & 054396 & 213.9870 & 52.2687 & & 0.66 & 0.10 & 100 & 0.3 & 43.80 & 7 & Type-1 AGN \\
\hline 2 & 067129 & 214.0352 & 52.3547 & $\ldots$ & 0.06 & 0.06 & 99 & 0.0 & 41.93 & 15 & NG hosting AGN \\
\hline 3 & 045621 & 214.0441 & 52.2727 & $\ldots$ & 0.25 & 0.27 & 98 & 0.9 & 43.39 & 19 & NG hosting AGN \\
\hline 4 & 068644 & 214.0572 & 52.3766 & $1.701(2)$ & 0.05 & 1.15 & 32 & 0.9 & 40.55 & 7 & Type-1 AGN \\
\hline 5 & 056094 & 214.0591 & 52.3276 & 0.534 (4) & 0.50 & 2.51 & 1 & 0.0 & 43.46 & 22 & NG hosting AGN \\
\hline 6 & 048319 & 214.0948 & 52.3212 & 1.603 (4) & 0.76 & 1.41 & 18 & 0.0 & 44.21 & 4 & SB-cont. AGN \\
\hline 7 & 019994 & 214.0956 & 52.2034 & $\ldots$ & 1.37 & 0.48 & 92 & 1.2 & 44.95 & 14 & Type-1 AGN \\
\hline 8 & 060727 & 214.1236 & 52.3925 & $\ldots$ & 0.96 & 0.59 & 81 & 0.3 & 44.40 & 10 & Type-2 AGN \\
\hline 9 & 053898 & 214.1298 & 52.3695 & $\ldots$ & 0.26 & 2.93 & 0 & 0.0 & 42.71 & 9 & Type-2 AGN \\
\hline 10 & 040342 & 214.1367 & 52.3171 & $1.028(4)$ & 0.95 & 0.83 & 60 & 0.0 & 43.81 & 4 & SB-cont. AGN \\
\hline 14 & 052726 & 214.1587 & 52.3857 & 0.417 (4) & 0.35 & 0.40 & 95 & 0.0 & 43.98 & 19 & NG hosting AGN \\
\hline 16 & 059064 & 214.1765 & 52.4241 & $\ldots$ & 0.05 & 2.26 & 1 & 0.3 & 43.89 & 15 & NG hosting AGN \\
\hline 17 & 029938 & 214.1768 & 52.3034 & $\ldots$ & 1.06 & 0.98 & 45 & 0.0 & 45.65 & 7 & Type-1 AGN \\
\hline 20 & 040860 & 214.1815 & 52.3506 & $0.283(2)$ & 0.12 & 0.91 & 53 & 0.0 & 43.20 & 20 & NG hosting AGN \\
\hline 21 & 045337 & 214.1832 & 52.3720 & $0.510(4)$ & 0.45 & 0.38 & 96 & 0.0 & 43.99 & 20 & NG hosting AGN \\
\hline 22 & 071927 & 214.1891 & 52.4850 & $1.630(2)$ & 1.55 & 0.24 & 99 & 0.9 & 44.08 & 14 & Type-1 AGN \\
\hline 24 & 054089 & 214.2060 & 52.4252 & $\ldots$ & 2.35 & 0.49 & 90 & 0.6 & 44.75 & 14 & Type-1 AGN \\
\hline 25 & 019616 & 214.2065 & 52.2815 & $0.761(4)$ & 0.75 & 0.62 & 78 & 0.3 & 43.78 & 22 & NG hosting AGN \\
\hline 26 & 024423 & 214.2079 & 52.3025 & 0.808 (4) & 0.73 & 0.23 & 99 & 0.0 & 43.87 & 3 & SB-dom. AGN \\
\hline 27 & 017652 & 214.2104 & 52.2763 & $0.683(4)$ & 0.60 & 0.29 & 98 & 0.9 & 44.16 & 6 & SB-cont. AGN \\
\hline 29 & 033772 & 214.2136 & 52.3461 & $\ldots$ & 0.85 & 1.67 & 7 & 0.0 & 44.70 & 9 & Type-2 AGN \\
\hline 30 & 058423 & 214.2163 & 52.4501 & $\ldots$ & 0.90 & 0.27 & 98 & 0.3 & 44.32 & 10 & Type-2 AGN \\
\hline 33 & 042611 & 214.2433 & 52.4036 & $\ldots$ & 0.90 & 0.87 & 53 & 0.0 & 42.61 & 4 & SB-cont. AGN \\
\hline 35 & 021276 & 214.2529 & 52.3218 & $\ldots$ & 0.32 & 0.15 & 100 & 0.3 & 43.61 & 9 & Type-2 AGN \\
\hline 36 & 041222 & 214.2675 & 52.4149 & $0.281(4)$ & 0.25 & 1.13 & 33 & 0.6 & 43.75 & 23 & NG hosting AGN \\
\hline 38 & 068074 & 214.2737 & 52.5297 & $0.426(2)$ & 1.56 & 1.59 & 9 & 0.0 & 44.13 & 2 & SB-dom. AGN \\
\hline 41 & 068708 & 214.2850 & 52.5403 & $\ldots$ & 1.36 & 0.62 & 80 & 0.3 & 44.16 & 5 & SB-dom. AGN \\
\hline 42 & 056274 & 214.2862 & 52.4917 & $\begin{array}{l}\cdots \\
\cdots\end{array}$ & 1.26 & 1.17 & 30 & 0.6 & 43.79 & 2 & SB-dom. AGN \\
\hline 43 & 046787 & 214.2870 & 52.4525 & $0.532(4)$ & 0.47 & 0.13 & 100 & 0.0 & 43.90 & 20 & NG hosting AGN \\
\hline 45 & 050845 & 214.2940 & 52.4747 & $\ldots$ & 1.25 & 0.99 & 45 & 0.3 & 44.53 & 1 & SB-dom. AGN \\
\hline 47 & 039386 & 214.2961 & 52.4280 & $\ldots$ & 0.34 & 0.93 & 51 & 0.3 & 44.00 & 19 & NG hosting AGN \\
\hline 48 & 062600 & 214.2984 & 52.5257 & $0.835(4)$ & 0.84 & 0.87 & 57 & 0.0 & 44.13 & 21 & NG hosting AGN \\
\hline 49 & 016716 & 214.2994 & 52.3366 & $0.433(4)$ & 0.45 & 1.37 & 18 & 0.6 & 44.08 & 22 & NG hosting AGN \\
\hline 50 & 036500 & 214.3096 & 52.4259 & $\ldots$ & 0.32 & 0.73 & 60 & 1.2 & 43.59 & 12 & Type-1 AGN \\
\hline 51 & 071816 & 214.3118 & 52.5720 & $\ldots$ & 1.20 & 1.38 & 18 & 1.2 & 44.17 & 2 & SB-dom. AGN \\
\hline 52 & 026610 & 214.3127 & 52.3869 & $1.271(3)$ & 0.48 & 0.27 & 99 & 0.0 & 43.41 & 14 & Type-1 AGN \\
\hline 53 & 041138 & 214.3134 & 52.4474 & 0.723 (4) & 0.67 & 1.08 & 38 & 0.0 & 44.14 & 9 & Type-2 AGN \\
\hline 55 & 041987 & 214.3290 & 52.4623 & $1.211(3)$ & 1.23 & 0.27 & 98 & 0.0 & 44.10 & 1 & SB-dom. AGN \\
\hline 56 & 042538 & 214.3303 & 52.4655 & $1.208(3)$ & 1.19 & 1.35 & 19 & 0.0 & 43.71 & 10 & Type-2 AGN \\
\hline 57 & 030161 & 214.3335 & 52.4168 & $\ldots$ & 0.88 & 1.03 & 42 & 0.3 & 44.19 & 8 & Type-2 AGN \\
\hline 59 & 055009 & 214.3456 & 52.5288 & $0.465(4)$ & 0.46 & 0.42 & 94 & 0.0 & 43.52 & 21 & NG hosting AGN \\
\hline 60 & 055370 & 214.3475 & 52.5316 & 0.484 (4) & 0.50 & 0.29 & 98 & 0.0 & 43.64 & 9 & Type-2 AGN \\
\hline 61 & 031265 & 214.3483 & 52.4320 & $\ldots$ & 1.19 & 1.01 & 43 & 1.2 & 44.42 & 1 & SB-dom. AGN \\
\hline 62 & 057218 & 214.3510 & 52.5416 & $0.902(4)$ & 0.83 & 1.39 & 17 & 0.0 & 43.59 & 3 & SB-dom. AGN \\
\hline 63 & 048619 & 214.3525 & 52.5069 & $0.482(4)$ & 0.54 & 1.50 & 12 & 0.3 & 44.49 & 14 & Type-1 AGN \\
\hline 64 & 069965 & 214.3553 & 52.5956 & $\ldots$ & 1.16 & 0.18 & 97 & 0.0 & 43.22 & 4 & SB-cont. AGN \\
\hline 66 & 051055 & 214.3637 & 52.5254 & $\ldots$ & 1.57 & 1.38 & 18 & 1.2 & 43.79 & 2 & SB-dom. AGN \\
\hline 67 & 068063 & 214.3704 & 52.5984 & $\ldots$ & 1.43 & 0.85 & 58 & 0.3 & 44.40 & 3 & SB-dom. AGN \\
\hline 69 & 034221 & 214.3748 & 52.4633 & $\ldots$ & 0.91 & 0.38 & 92 & 0.6 & 44.50 & 10 & Type-2 AGN \\
\hline 72 & 035715 & 214.3784 & 52.4718 & $\ldots$ & 1.42 & 1.19 & 29 & 1.2 & 43.79 & 2 & SB-dom. AGN \\
\hline 73 & 049420 & 214.3859 & 52.5342 & $0.986(4)$ & 0.91 & 0.38 & 96 & 0.6 & 43.87 & 16 & NG hosting AGN \\
\hline 74 & 055653 & 214.3909 & 52.5637 & $0.551(4)$ & 0.52 & 0.39 & 95 & 0.3 & 43.90 & 22 & NG hosting AGN \\
\hline 75 & 019988 & 214.3911 & 52.4155 & $\ldots$ & 0.92 & 1.14 & 33 & 0.0 & 43.72 & 3 & SB-dom. AGN \\
\hline 76 & 044463 & 214.3932 & 52.5186 & $0.271(4)$ & 0.29 & 0.42 & 95 & 0.6 & 43.86 & 16 & NG hosting AGN \\
\hline 77 & 032243 & 214.3952 & 52.4696 & $\ldots$ & 1.73 & 1.52 & 11 & 0.0 & 45.29 & 1 & SB-dom. AGN \\
\hline 78 & 040934 & 214.3998 & 52.5083 & $\cdots$ & 2.42 & 0.61 & 82 & 0.3 & 45.61 & 14 & Type-1 AGN \\
\hline 79 & 039818 & 214.4012 & 52.5047 & $\ldots$ & 0.93 & 1.31 & 21 & 0.3 & 42.85 & 2 & SB-dom. AGN \\
\hline 80 & 061825 & 214.4014 & 52.5957 & $0.197(1)$ & 2.32 & 1.56 & 10 & 0.0 & 44.92 & 1 & SB-dom. AGN \\
\hline 81 & 016037 & 214.4037 & 52.4084 & .. & 2.33 & 1.37 & 19 & 0.3 & 44.79 & 14 & Type-1 AGN \\
\hline 82 & 062180 & 214.4043 & 52.5994 & $\ldots$ & 0.25 & 1.22 & 27 & 0.0 & 44.79 & 22 & NG hosting AGN \\
\hline 83 & 035272 & 214.4056 & 52.4893 & $\ldots$ & 1.19 & 0.36 & 87 & 0.9 & 43.83 & 9 & Type-2 AGN \\
\hline 84 & 053837 & 214.4112 & 52.5706 & $\ldots$ & 1.11 & 1.17 & 30 & 0.3 & 43.65 & 2 & SB-dom. AGN \\
\hline 86 & 031503 & 214.4127 & 52.4789 & $\cdots$ & 0.90 & 0.74 & 69 & 1.2 & 43.68 & 9 & Type-2 AGN \\
\hline 87 & 031796 & 214.4137 & 52.4806 & $\ldots$ & 1.02 & 0.53 & 88 & 0.0 & 44.32 & 10 & Type-2 AGN \\
\hline 89 & 057956 & 214.4228 & 52.5959 & $\ldots$ & 1.39 & 0.37 & 95 & 0.0 & 44.33 & 10 & Type-2 AGN \\
\hline
\end{tabular}


Table 1

(Continued)

\begin{tabular}{|c|c|c|c|c|c|c|c|c|c|c|c|}
\hline ID & ID IRAC & R.A. $\left(^{\circ}\right)$ & Decl. $\left({ }^{\circ}\right)$ & $z_{\text {spec }}$ & $z_{\text {phot }}$ & $\chi_{v}^{2}$ & Prob $(\%)$ & $A_{V}$ & $L_{r}$ & Template & Group \\
\hline 90 & 028146 & 214.4244 & 52.4732 & $1.148(4)$ & 1.15 & 2.43 & 1 & 0.6 & 44.53 & 4 & SB-cont. AGN \\
\hline 91 & 031444 & 214.4393 & 52.4976 & $0.873(4)$ & 0.87 & 1.27 & 24 & 0.9 & 43.76 & 1 & SB-dom. AGN \\
\hline 92 & 024070 & 214.4401 & 52.4672 & $0.224(2)$ & 0.23 & 0.61 & 82 & 0.0 & 43.03 & 22 & NG hosting AGN \\
\hline 93 & 033761 & 214.4415 & 52.5091 & $0.985(3)$ & 0.97 & 1.95 & 3 & 0.6 & 44.43 & 8 & Type-2 AGN \\
\hline 95 & 027043 & 214.4445 & 52.4829 & $\ldots$ & 1.78 & 0.93 & 50 & 0.9 & 43.90 & 2 & SB-dom. AGN \\
\hline 97 & 024055 & 214.4460 & 52.4713 & $\ldots$ & 2.58 & 0.27 & 90 & 1.2 & 43.83 & 12 & Type-1 AGN \\
\hline 98 & 051437 & 214.4472 & 52.5862 & $1.547(3)$ & 2.37 & 0.92 & 51 & 0.3 & 43.81 & 7 & Type-1 AGN \\
\hline 99 & 021585 & 214.4550 & 52.4676 & $0.996(4)$ & 1.00 & 0.50 & 87 & 0.3 & 44.65 & 17 & NG hosting AGN \\
\hline 101 & 035904 & 214.4575 & 52.5290 & $\ldots$ & 2.65 & 0.47 & 88 & 0.9 & 44.30 & 2 & SB-dom. AGN \\
\hline 105 & 030608 & 214.4657 & 52.5129 & $\ldots$ & 0.87 & 0.58 & 82 & 0.3 & 43.69 & 10 & Type-2 AGN \\
\hline 106 & 022680 & 214.4684 & 52.4814 & $\ldots$ & 1.00 & 0.57 & 84 & 0.6 & 43.92 & 9 & Type-2 AGN \\
\hline 107 & 021273 & 214.4707 & 52.4775 & $0.671(3)$ & 0.60 & 0.40 & 95 & 0.3 & 43.96 & 22 & NG hosting AGN \\
\hline 108 & 045400 & 214.4737 & 52.5795 & $0.719(4)$ & 0.65 & 0.51 & 88 & 0.6 & 43.77 & 9 & Type-2 AGN \\
\hline 109 & 028312 & 214.4748 & 52.5095 & $\ldots$ & 1.34 & 1.06 & 39 & 0.3 & 44.43 & 8 & Type-2 AGN \\
\hline 110 & 031338 & 214.4760 & 52.5232 & $\ldots$ & 0.65 & 0.81 & 61 & 1.2 & 43.45 & 10 & Type-2 AGN \\
\hline 112 & 047305 & 214.4803 & 52.5924 & $\ldots$ & 2.75 & 0.34 & 96 & 0.3 & 43.91 & 12 & Type-1 AGN \\
\hline 113 & 029613 & 214.4868 & 52.5235 & $\ldots$ & 0.50 & 0.52 & 88 & 1.2 & 42.79 & 7 & Type-1 AGN \\
\hline 116 & 027980 & 214.4893 & 52.5186 & $\ldots$ & 0.16 & 0.70 & 67 & 0.9 & 42.79 & 7 & Type-1 AGN \\
\hline 118 & 029054 & 214.4956 & 52.5275 & $\ldots$ & 0.63 & 0.15 & 100 & 0.6 & 43.69 & 9 & Type-2 AGN \\
\hline 119 & 046309 & 214.5015 & 52.6030 & $\ldots$ & 2.45 & 0.31 & 93 & 0.0 & 43.69 & 13 & SB-cont. AGN \\
\hline 124 & 041429 & 214.5082 & 52.5875 & $\ldots$ & 1.33 & 0.19 & 100 & 0.6 & 45.13 & 14 & Type-1 AGN \\
\hline 125 & 042989 & 214.5119 & 52.5965 & $\ldots$ & 2.05 & 1.14 & 33 & 0.3 & 43.45 & 1 & SB-dom. AGN \\
\hline 126 & 044785 & 214.5191 & 52.6092 & $0.387(1)$ & 1.61 & 1.88 & 4 & 1.2 & 44.49 & 7 & Type-1 AGN \\
\hline 127 & 032921 & 214.5270 & 52.5662 & $\ldots$ & 1.30 & 0.70 & 71 & 0.0 & 45.05 & 10 & Type-2 AGN \\
\hline 128 & 018428 & 214.5305 & 52.5083 & $\ldots$ & 0.90 & 0.57 & 84 & 0.0 & 44.35 & 20 & NG hosting AGN \\
\hline 133 & 024215 & 214.5679 & 52.5586 & $\ldots$ & 2.38 & 2.28 & 1 & 0.6 & 44.54 & 2 & SB-dom. AGN \\
\hline 134 & 016978 & 214.5751 & 52.5340 & $\ldots$ & 1.32 & 2.04 & 3 & 0.3 & 43.89 & 3 & SB-dom. AGN \\
\hline 135 & 022888 & 214.5841 & 52.5647 & $\ldots$ & 0.25 & 1.36 & 19 & 0.6 & 42.35 & 7 & Type-1 AGN \\
\hline 136 & 018192 & 214.5888 & 52.5485 & $0.036(1)$ & 0.05 & 0.59 & 81 & 1.2 & 40.70 & 7 & Type-1 AGN \\
\hline
\end{tabular}

Notes. Templates: 1, 2-Starburst/ULIRG, 3, 5-Starburst, 4-Sy1/Starburst/ULIRG, 6-Sy2/Starburst, 13-Sy2/Starburst/ULIRG, 7, 12, 14-Type-1 QSO, 8-Type-2 QSO, 9-Sy1.8, 10-Sy2, 11-Torus-QSO, 15, 16, 17-Ellipticals of 2, 5, and 13 Gyr, 18, 19, 20, 21, 22, 23-Spirals of types S0, Sa, Sb, Sc, Sd, and Sdm.

Data presented: ID from Barmby et al. (2006), IRAC ID, IRAC $3.6 \mu \mathrm{m}$ J2000.0 right ascension and declination, spectroscopic redshift from DEEP public database with its corresponding reliability between brackets $(1-2=$ low reliability, $3-4=$ high reliability), photometric redshift and its corresponding $\chi_{v}^{2}$ and probability, optical extinction derived from the Calzetti et al. (2000) reddening law, logarithm of $v L_{v}$ in the $r$ band as a reference, in erg s ${ }^{-1}$, fitted template, and main group classification.

from the total sample. Considering only the 31 objects with highly reliable $z_{\text {spec }}$, there are 4 Starburst-dominated AGNs, 4 Starburst-contaminated AGNs, 3 Type-1 AGNs, 5 Type-2 AGNs, and 15 Normal galaxy hosting AGNs. Thus, it is very difficult to check the redshift failure rate for the different groups. Only for the Normal galaxy hosting AGNs we can confirm the success in the redshift determination with this set of templates, since $\sim 50 \%$ of the total number of objects fitted with elliptical or spiral templates have $z_{\text {spec }}$ to compare with. Due to the flat and featureless SED typical of Type-1 QSOs, the Type-1 AGNs group of templates could produce the less reliable photometric redshifts of the sample (Franceschini et al. 2005). We cannot then discard that any subset of templates produces higher redshift failure rates than others, but looking at the distribution of the objects belonging to the different groups of AGN in the various diagnostic diagrams in the following sections, and at the correlations displayed by them, we are confident that our SED classification and redshift determination are as good for the rest of the groups as they are for Normal galaxy hosting AGN.

\section{DISCUSSION}

\subsection{Classification by SEDs and Photometric Redshift Distribution of the Sample}

Together with the photometric redshift calculations reported in the previous section, we obtain SED fits, that allow us to distinguish between different types of AGN populations, i.e., whether they are pure AGNs, AGNs hosted by starburstdominated galaxies, or AGNs in otherwise normal galaxies.

For the five main groups described before we obtain the following distribution: Starburst-dominated AGN (24\% of the sample), Starburst-contaminated AGN (7\%), Type-1 AGN (21\%), Type-2 AGN (24\%), and Normal galaxy hosting AGN (24\%).

We consider the Type-1 AGNs, Type-2 AGNs, and Starburst-contaminated AGNs as representative groups of AGN-dominated galaxies (since their SEDs are AGN-like at all or almost all wavelength ranges). The Starburst-dominated AGNs and Normal galaxy hosting AGNs are likewise considered AGNs somehow masked by their host emission. With this simple classification, we find that $52 \%$ of the sample is 

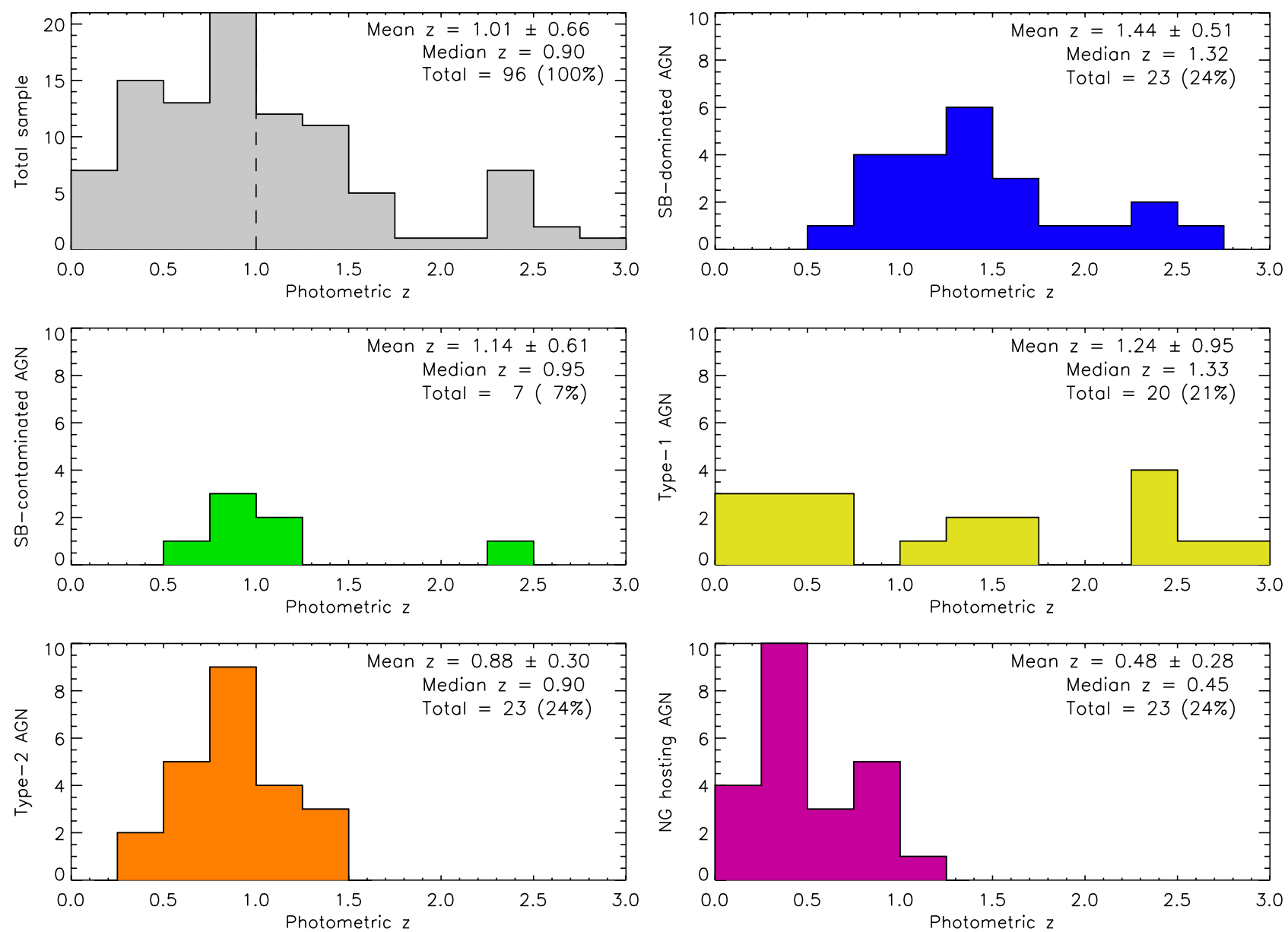

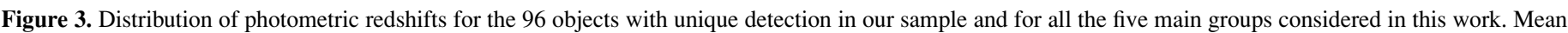

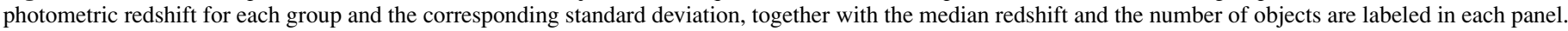
(A color version of this figure is available in the online journal.)

AGN-dominated while $48 \%$ is host-galaxy-dominated, i.e., half of the objects in the EGS sample of AGNs show AGN-like SEDs while the other half show host-dominated SEDs. This is consistent with the finding that between $40 \%$ and $60 \%$ of the Chandra selected galaxies in the Hawaii Deep Survey Field SSA13 and in the Chandra Deep Field North (Barger et al. 2001 and Hornschemeier et al. 2001, respectively) have optical spectra with no signs of nuclear activity.

Also Barmby et al. (2006), based on the IRAC slopes $(\alpha<0$ for the red power-law IRAC SEDs, and $\alpha>0$ for the blue ones) divided their sample into sources where the central engine dominates the IRAC SEDs and stellar-dominated galaxies. They found that $40 \%$ of the sources have red power-law SEDs, another $40 \%$ have blue host-dominated mid-IR SEDs, and the remaining $20 \%$ could not be fitted with a power-law.

The method employed in this paper constitutes a powerful technique of classification of high redshift AGNs provided we are able to procure well sampled SEDs. This is important, for instance, for multiband deep surveys of galaxies for which spectroscopic data will be necessarily scarce. Having SEDs over the largest wavelength range as possible is mandatory to identify the entire AGN population (Dye 2008). Otherwise, depending on the observed wavelength, the galaxies could be misclassified. This is crucial, for example, for our Starburstcontaminated AGNs, that in the optical range look like starburst galaxies, and toward redder wavelengths appear as Type-1 or Type-2 AGNs. Dye (2008) also finds that the results of the SED fitting show little difference between two filter sets that span the same wavelength range, despite the number of filters used. Nevertheless, from our work, we find that including a large number of filters can reveal details in the SED shape that help the code choose between different templates. This is crucial to distinguish among the different templates of a given group, for which little differences in the SED determine the type of object, or its age (Polletta et al. 2007).

We use now the classification of the galaxies obtained to investigate the properties of the different AGN groups. The distribution of redshifts for all the 96 objects with unique detection in our sample is shown in the top-left panel of Figure 3. Fifty-eight percent of the sample has $z<1$, with the rest of the sources distributed in a decreasing tail up to $z=3$. This is expected for X-ray selected samples with similar or even deeper flux limits (Hasinger 2003; Barger et al. 2005). Figure 3 also shows histograms for the photometric redshift distributions of the Starburst-dominated AGNs, Starburstcontaminated AGNs, Type-1 AGNs, Type-2 AGNs, and the Normal galaxy hosting AGNs groups.

Type-2 AGNs and Normal galaxy hosting AGNs are concentrated at lower redshifts, whereas the Starburst-dominated AGNs show a high concentration around $z_{\text {phot }}=1.3$. The Type-1 AGNs group has the largest spread in redshift, its mean value being $z_{\text {phot }}=1.24 \pm 0.95$. The Starburst-contaminated AGNs group contains only seven objects, six of them within the redshift range $[0.6,1.2]$, the other having a $z_{\text {phot }}=2.45$. 
This indicates that the Starburst-dominated AGNs constitute the high-redshift population of AGNs masked by powerful host emission, while the Normal galaxy hosting AGNs group represents the low-redshift population of low-luminosity AGNs also masked by their host galaxies. Previous studies suggest that most low luminosity AGNs are found in massive, mostly spheroidal galaxies (Dunlop et al. 2003; Kauffmann et al. 2003; Grogin et al. 2005; Pierce et al. 2007). Something similar happens with the AGN-dominated group: the Type-1 AGNs span a large redshift distribution, the Starburst-contaminated AGNs are located at intermediate values of redshift, and finally, the Type-2 AGNs are the low- $z$ objects in this subsample.

Alonso-Herrero et al. (2004) found that $\sim 25 \%$ of their X-ray and $24 \mu \mathrm{m}$ selected sources in both the EGS and the Lockman Hole (45 in total) show pure Type-1 AGN SEDs, while more than half of the sample have stellar emission-dominated or obscured SEDs. Franceschini et al. (2005) detected 99 AGN in the $\mathrm{X}$-rays and mid-IR with Spitzer in the SWIRE survey (Lonsdale et al. 2003), sorting them in three main groups: Type-1 AGN (39\%), Type-2 AGN (23\%), and normal and starburst galaxies (38\%). By adding Piccinotti et al. (1982) and Kuraszkiewicz et al. (2003) samples, there are 32 AGNs with $z \leqslant 0.12$, also selected both in the hard X-rays and mid-IR, with more than half of these sources being Type-1 AGN according to their SEDs. Ours and other works (e.g., Alonso-Herrero et al. 2004; Franceschini et al. 2005) performed with SED classification of $\mathrm{X}$-ray and mid-IR selected AGN in a wide range of redshift (up to $z \sim 2-3$ ), when compared with the results obtained for local samples of AGNs selected in the same bands, seem to indicate that the percentage of Type-1 objects decreases with redshift, while the number of obscured AGNs at high redshift increases.

Although the data used in this paper do not allow a deep study of the AGN feedback phenomenon, it is worth noting that a redshift sequence can be readily seen in Figure 3. Indeed, the Starburst-dominated AGNs would have the highest redshifts in a decreasing sequence that goes through the Type-1 AGNs, Starburst-contaminated AGNs, and Type-2 AGNs, ending with the Normal galaxy hosting AGNs group that shows the lowest redshifts. This evolutionary sequence has been noticed for earlytype galaxies by Schawinski et al. (2007). According to this recent work, the starbursts would start and be the dominant player after its onset. Subsequently, as the $\mathrm{BH}$ accretes enough mass, the AGN feedback reveals itself as the $\mathrm{BH}$ competes for the gas reservoir with the starbursts, eventually quenching the star formation. The starburst phase thus declines, the AGN becoming dominant. The Starburst-contaminated AGNs phase would be the transition phase mentioned by Schawinski et al. (2007). This process continue through lower ionization phases and it will end with the more quiescent Normal galaxy hosting AGNs phases at lower redshifts.

\subsection{Correlations}

\subsubsection{Correlations for the Whole Sample}

One of the main advantages of the sample we are discussing is the multiwavelength coverage of the data, which allows us to study for the first time various correlations between UV/optical/ IR luminosities and X-ray luminosities for such a big AGN sample and within this range of redshift. The aim is to understand the behavior of these sources in the different wavelength ranges.

Absolute magnitudes $\left(M_{\mathrm{ABS}}\right)$ computed by HyperZ in each filter using the photometric redshifts and the chosen cosmological parameters are used here to derive luminosities for the
96 objects with unique detection. The HyperZ code provides the $M_{\mathrm{ABS}}$ (including the $K$-correction) in the UV, optical, NIR, and mid-IR filters considered. Regarding the X-ray data, the observed rest-frame hard and soft X-ray luminosities are obtained from the equation $L_{X}=4 \pi d_{L}^{2} f_{X} /(z+1)^{2-\Gamma}$, where $d_{L}$ is the luminosity distance $(\mathrm{cm}), \mathrm{f}_{X}$ is the X-ray flux $\left(\mathrm{ergs} \mathrm{cm}^{-2}\right.$ $\mathrm{s}^{-1}$ ), and $\Gamma$ is the photon index. In this case, the $K$-correction vanishes since we assume a photon index $\Gamma=2$ (Krumpe et al. 2007; Alexander et al. 2003; Mainieri et al. 2002), which is the canonical value for unobscured AGNs (George et al. 2000). Obscured active nuclei have considerably flatter effective $\mathrm{X}$-ray spectral slopes, due to the energy-dependent photoelectric absorption of the X-ray emission (Risaliti et al. 1999). However, Mainieri et al. (2002) find the same intrinsic slope of the X-ray spectrum for both Type-1 and Type-2 AGN whatever their absorption levels, with $\Gamma \sim 2$ for an X-ray-selected sample in the Lockman Hole. We therefore assume a photon index $\Gamma=2$ for both obscured and unobscured AGNs, and consequently no $K$-correction is needed for the $\mathrm{X}$-ray luminosities.

The first row of Table 2 shows the fitting slopes and correlation coefficients ( $r$ ) of each scatter diagram between the FUV/NUV/ ugriz/JK/IRAC/MIPS luminosities and the hard/soft X-ray luminosities for the fits including all the objects with unique detection. In all the cases Spearman's rank correlation test has been performed, confirming that all the correlations are significant $(p<0.01)$. Examples of these correlations for the $\mathrm{FUV} / \mathrm{NUV} / r / K /$ IRAC $4.5 \mu \mathrm{m} / \mathrm{MIPS} 24 \mu \mathrm{m}$ luminosities and the hard/soft X-ray luminosities are shown in Figure 4.

The expected slopes for AGN-dominated objects should be close to unity, since if the active nucleus is the dominant emitting source at all wavelengths, tight linear correlations should be drawn. Reality is different, and AGNs are actually hosted by different types of galaxies. As it has been seen in previous sections, these host galaxies contaminate or even mask the AGN emission, thus deviating correlations from linear and worsening them. Both the X-ray and mid-IR emissions are mostly dominated by the active nuclei, whereas the optical and, to a lesser extent, the NIR bands are more affected by extinction, by stellar emission from the host galaxy, or by both. This is clearly reflected in the slopes and correlation coefficients (hereafter $\alpha$ and $r$ ) of the global fits (see first row of Table 2). Although correlations are all significant, with both the slopes and correlation coefficients close to unity, they begin getting slightly blurred as wavelength increases from the bluest optical bands up to the $K$ band, improving again in the mid-IR. The blurring is more noticeable when soft instead of hard X-rays are considered, due to the higher obscuration that affects the lower energies.

Correlations between UV and X-ray luminosities are also good. The slopes are $\alpha \sim 1.2$ and 1.1 for the FUV versus both the hard and soft X-rays luminosities, respectively, in good agreement with early X-ray studies of AGN that find correlations between X-ray and UV monochromatic luminosities with similar slopes: $L_{X} \propto L_{U V}^{\beta}$, with $\beta \sim 0.7-0.8$, thus $\alpha \sim 1.4-1.2$ (Wilkes et al. 1994; Vignali et al. 2003; Strateva et al. 2005; Steffen et al. 2006). Nevertheless, this range of $\alpha$ was determined by using $2 \mathrm{keV}$ and $2500 \AA$ luminosities, which correspond to soft X-rays and NUV, respectively. The slopes measured by us for the NUV versus both the hard and soft X-rays luminosities are $\alpha \sim 0.8$ and 0.7, respectively, which are lower than expected. Nevertheless, LaFranca et al. (1995) found a correlation consistent with $\alpha=1$ using a generalized orthogonal regression that is in better agreement with our values. 
Table 2

Fitting Slopes and Correlation Coefficients Corresponding to All Considered Luminosity-Luminosity Scatter Diagrams

\begin{tabular}{|c|c|c|c|c|c|c|c|c|c|c|c|c|}
\hline \multirow[t]{2}{*}{ Band } & \multicolumn{2}{|c|}{ Total Fit } & \multicolumn{2}{|c|}{ SB-Dom. AGN } & \multicolumn{2}{|c|}{ SB-Cont. AGN } & \multicolumn{2}{|c|}{ Type-1 AGN } & \multicolumn{2}{|c|}{ Type-2 AGN } & \multicolumn{2}{|c|}{ NG Hosting AGN } \\
\hline & $\alpha$ & $r$ & $\alpha$ & $r$ & $\alpha$ & $r$ & $\alpha$ & $r$ & $\alpha$ & $r$ & $\alpha$ & $r$ \\
\hline FUV & 1.22 & 0.84 & 1.05 & 0.81 & 1.37 & 0.94 & 1.29 & 0.95 & $\ldots$ & $\ldots$ & 1.12 & 0.71 \\
\hline NUV & 0.83 & 0.73 & 1.08 & 0.83 & 0.87 & 0.87 & 0.99 & 0.85 & $\ldots$ & $\ldots$ & 0.72 & 0.52 \\
\hline$u$ & 0.75 & 0.73 & 0.90 & 0.77 & $\ldots$ & $\ldots$ & 1.01 & 0.89 & $\ldots$ & $\ldots$ & $\ldots$ & $\ldots$ \\
\hline$g$ & 0.70 & 0.72 & 0.79 & 0.72 & 1.12 & 0.80 & 0.99 & 0.91 & 0.76 & 0.58 & $\ldots$ & $\ldots$ \\
\hline$r$ & 0.67 & 0.71 & 0.71 & 0.66 & $\ldots$ & $\ldots$ & 0.97 & 0.92 & 0.71 & 0.57 & $\ldots$ & $\ldots$ \\
\hline$i$ & 0.66 & 0.73 & 0.71 & 0.66 & $\ldots$ & $\ldots$ & 0.96 & 0.93 & 0.64 & 0.55 & 0.47 & 0.58 \\
\hline$z$ & 0.67 & 0.73 & 0.78 & 0.74 & $\ldots$ & $\ldots$ & 0.97 & 0.93 & 0.64 & 0.59 & 0.48 & 0.57 \\
\hline$J$ & 0.68 & 0.75 & 0.82 & 0.79 & $\ldots$ & $\ldots$ & 0.97 & 0.94 & 0.75 & 0.62 & 0.48 & 0.58 \\
\hline$K$ & 0.72 & 0.78 & 0.78 & 0.82 & 1.25 & 0.87 & 0.91 & 0.92 & 0.66 & 0.51 & 0.62 & 0.67 \\
\hline IRAC3.6 & 0.81 & 0.80 & 1.01 & 0.83 & 1.40 & 0.88 & 0.90 & 0.88 & 0.67 & 0.49 & 0.72 & 0.66 \\
\hline IRAC4.5 & 0.87 & 0.80 & 1.07 & 0.82 & $\ldots$ & $\ldots$ & 0.90 & 0.86 & 0.77 & 0.51 & 0.82 & 0.67 \\
\hline IRAC5.8 & 0.91 & 0.79 & 0.96 & 0.73 & 1.38 & 0.84 & 0.90 & 0.85 & 0.81 & 0.53 & 0.88 & 0.65 \\
\hline IRAC8.0 & 0.93 & 0.80 & 0.69 & 0.64 & 1.40 & 0.85 & 0.92 & 0.87 & 0.82 & 0.56 & 0.98 & 0.69 \\
\hline MIPS24 & 1.02 & 0.70 & $\ldots$ & $\ldots$ & $\ldots$ & $\ldots$ & 0.89 & 0.84 & 0.87 & 0.50 & 1.23 & 0.67 \\
\hline \multirow[t]{2}{*}{ Band } & \multicolumn{2}{|c|}{ Total Fit } & \multicolumn{2}{|c|}{ SB-Dom. AGN } & \multicolumn{2}{|c|}{ SB-Cont. AGN } & \multicolumn{2}{|c|}{ Type-1 AGN } & \multicolumn{2}{|c|}{ Type-2 AGN } & \multicolumn{2}{|c|}{ NG Hosting AGN } \\
\hline & $\alpha$ & $r$ & $\alpha$ & $r$ & $\alpha$ & $r$ & $\alpha$ & $r$ & $\alpha$ & $r$ & $\alpha$ & $r$ \\
\hline FUV & 1.07 & 0.83 & 0.92 & 0.80 & 0.92 & 0.95 & 1.17 & 0.95 & $\ldots$ & $\ldots$ & 0.92 & 0.63 \\
\hline NUV & 0.75 & 0.74 & 0.87 & 0.85 & $\ldots$ & $\ldots$ & 0.87 & 0.86 & $\ldots$ & $\ldots$ & 0.68 & 0.54 \\
\hline$u$ & 0.66 & 0.73 & 0.70 & 0.78 & $\ldots$ & $\ldots$ & 0.87 & 0.89 & $\ldots$ & $\ldots$ & $\ldots$ & $\ldots$ \\
\hline$g$ & 0.61 & 0.71 & 0.64 & 0.72 & $\ldots$ & $\ldots$ & 0.85 & 0.90 & $\ldots$ & $\ldots$ & $\ldots$ & $\ldots$ \\
\hline$r$ & 0.58 & 0.70 & $\ldots$ & $\ldots$ & $\ldots$ & $\ldots$ & 0.84 & 0.90 & $\ldots$ & $\ldots$ & $\ldots$ & $\ldots$ \\
\hline$i$ & 0.57 & 0.70 & $\ldots$ & $\ldots$ & $\ldots$ & $\ldots$ & 0.83 & 0.91 & $\ldots$ & $\ldots$ & 0.44 & 0.58 \\
\hline$z$ & 0.57 & 0.70 & $\ldots$ & $\ldots$ & $\ldots$ & $\ldots$ & 0.83 & 0.91 & $\ldots$ & $\ldots$ & 0.45 & 0.59 \\
\hline$J$ & 0.59 & 0.72 & 0.52 & 0.65 & $\ldots$ & $\ldots$ & 0.82 & 0.91 & $\ldots$ & $\ldots$ & 0.45 & 0.59 \\
\hline$K$ & 0.62 & 0.76 & 0.49 & 0.67 & $\ldots$ & $\ldots$ & 0.79 & 0.91 & $\ldots$ & $\ldots$ & 0.55 & 0.65 \\
\hline IRAC3.6 & 0.70 & 0.79 & 0.64 & 0.71 & $\ldots$ & $\ldots$ & 0.79 & 0.88 & $\ldots$ & $\ldots$ & 0.64 & 0.65 \\
\hline IRAC4.5 & 0.76 & 0.79 & 0.73 & 0.74 & $\ldots$ & $\ldots$ & 0.80 & 0.87 & $\ldots$ & $\ldots$ & 0.71 & 0.65 \\
\hline IRAC5.8 & 0.79 & 0.77 & 0.72 & 0.72 & $\ldots$ & $\ldots$ & 0.81 & 0.87 & $\ldots$ & $\ldots$ & 0.76 & 0.62 \\
\hline IRAC8.0 & 0.80 & 0.77 & 0.58 & 0.67 & $\ldots$ & $\ldots$ & 0.81 & 0.88 & $\ldots$ & $\ldots$ & 0.81 & 0.63 \\
\hline MIPS24 & 0.87 & 0.68 & 0.66 & 0.61 & $\ldots$ & $\ldots$ & 0.77 & 0.84 & $\ldots$ & $\ldots$ & 1.02 & 0.61 \\
\hline
\end{tabular}

Notes. Spearman's rank correlation tests have been performed for all scatter diagrams. The values reported in Table 2 are indeed significant $(p<0.01)$.

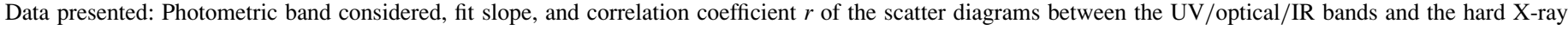

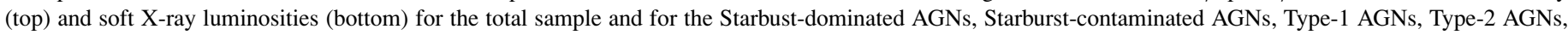
and Normal galaxy hosting AGNs groups.

\subsubsection{Correlations for the Main AGN Groups}

We also report slopes and correlation coefficients for the five main groups considered in the last sections separately in Table 2. Spearman's rank correlation tests have been performed for all scatter diagrams. Thus, the values reported in Table 2 correspond only to objects showing significant correlations $(p<0.01)$.

Looking at the Type-1 AGNs in Table 2 we conclude that they are the less contaminated active nuclei of the sample. Since we are seeing a direct view of the central engine, the emission is dominated by the AGNs at all wavelengths. Therefore, these objects draw the most tight correlations between each photometric band and either the hard or the soft X-ray luminosities. ${ }^{5}$

If we look at the Starburst-contaminated AGNs, we find significant correlations between the hard X-rays and the UV and IR bands, disappearing for the soft X-rays, due to the higher obscuration in this wavelength range.

\footnotetext{
5 Note that in order to check that the good correlation displayed by Type-1 AGNs luminosities is not due to a distance effect (this group of galaxies presents the largest spread in redshift, as shown in Figure 3), we have also analyzed the corresponding fluxes, instead of luminosities, for all the groups considered in this section. This way, the distance effect is eliminated from the fits. We find the same linear and significant correlations for Type-1 AGNs fluxes, confirming that the correlations displayed for this group of AGN are only due to their intrinsic properties.
}

For the Starburst-dominated AGNs, correlations including the hard X-ray data are better and more robust than those with the soft X-ray ones, for which both the slope and $r$ values are far from unity. This is certainly due to the higher obscuration affecting the soft X-ray emission in these objects. The host galaxy emission and the dust are indeed masking the AGN. The same thing happens, but more dramatically, with the Type-2 AGNs group, for which all the correlations involving the soft $\mathrm{X}$-ray emission are not significant.

It is worth mentioning the worsening of the fits for the Starburst-dominated AGNs when the hard X-ray and either the IRAC $8 \mu \mathrm{m}$ or MIPS $24 \mu \mathrm{m}$ emission are considered. The slopes and correlation coefficients of both fits move away from unity, something that is interpreted as being due to the increasing importance of the starburst emission at these longer wavelengths. If we look at the MIPS $24 \mu \mathrm{m}$ luminosityluminosity scatter diagrams (bottom of Figure 4), the overall majority of the Starburst-dominated AGNs are located above the fit line. This indicates that there is an excess of mid-IR emission, as compared with the $\mathrm{X}$-ray luminosity coming principally from the AGN. This mid-IR excess comes from warm dust heated by the intense star formation bursts taking place in the galaxy (in addition to the dust heated by the AGN), hence deviating the 


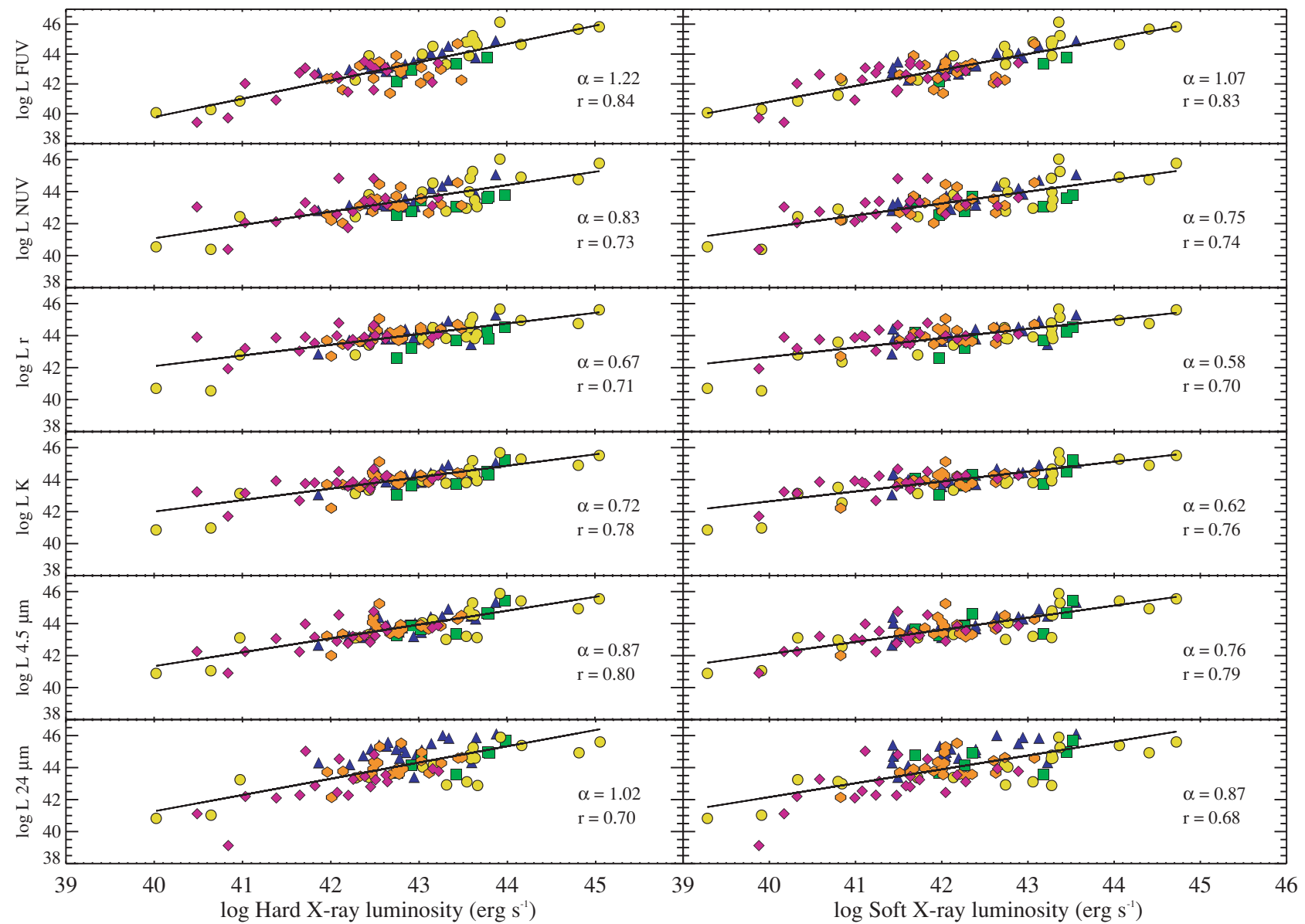

Figure 4. Examples of luminosity-luminosity scatter diagrams for all the objects in our sample with unique detections and published data in the considered bands. Logarithms of $\nu L_{v}$ in the FUV, NUV, $r, K$, IRAC $4.5 \mu \mathrm{m}$, and MIPS $24 \mu \mathrm{m}$ bands (erg s ${ }^{-1}$ ) are represented vs. their hard and soft X-ray counterparts. Symbols are the same as in Figure 2, indicating the template fitting classification.

(A color version of this figure is available in the online journal.)

Table 3

Hard and Soft X-ray Luminosity Ranges (log) for the Starburst-dominated AGNs, Starburst-contaminated AGNs, Type-1 AGNs, Type-2 AGNs, and Normal galaxy hosting AGNs Groups

\begin{tabular}{lccccc}
\hline \hline X-Ray Range & SB-Dom. AGN & SB-Cont. AGN & Type-1 AGN & Type-2 AGN & NG Hosting AGN \\
\hline Hard & {$[42,44]$} & {$[43,44]$} & {$[40,45]$} & {$[42,43]$} & {$[40,43]$} \\
Soft & {$[41,44]$} & {$[42,44]$} & {$[39,45]$} & {$[41,43]$} & {$[40,43]$} \\
\hline
\end{tabular}

Note. Luminosities are not corrected for absorption.

Starburst-dominated AGNs group from the linear fit, and making the correlation nonsignificant when the MIPS $24 \mu$ m luminosity is considered.

The behavior of Normal galaxy hosting AGNs is completely different: correlations when either the soft or hard X-rays are considered are quite similar, improving toward longer wavelengths, where the AGN resurfaces. This group of galaxies include low-luminosity AGNs hosted in normal galaxies that dominates the optical and NIR bands, but not the mid-IR emission. This explains why in some fits performed with this subset of templates, the IRAC 8 and MIPS $24 \mu \mathrm{m}$ are not completely well reproduced by the fit.

\subsection{X-Ray Properties}

Looking at the hard and soft luminosity ranges (see Table 3 ) for each of the five main groups described above, we find that Type-1 AGNs present the largest spread in luminosity, together with the highest luminosity values in both bands
$\left(L_{\text {Hard }}=10^{40-45}\right.$ and $L_{\text {Soft }}=10^{39-45} \mathrm{erg} \mathrm{s}^{-1}$, not corrected for absorption). Alonso-Herrero et al. (2006) found that the majority of galaxies in their sample of X-ray detected sources in the Chandra Deep Field-South fitted with broad-line AGN (BLAGN) QSO templates showed hard X-ray luminosities in the range $10^{43-44} \mathrm{erg} \mathrm{s}^{-1}$ (also not corrected for absorption). The same has been found when a spectroscopic classification of the objects has been possible (Zheng et al. 2004; Szokoly et al. 2004; Barger et al. 2005). Our hard X-ray luminosity range for Type-1 AGNs agrees with the literature in the sense that the most luminous $\mathrm{X}$-ray sources are enclosed in that range, while five sources show $L_{\mathrm{Hard}}<10^{43} \mathrm{erg} \mathrm{s}^{-1}$, and only three have $L_{\mathrm{Hard}}<10^{42} \mathrm{erg} \mathrm{s}^{-1}$, namely, irac068644, irac027980, and irac018192, all of them with $z_{\text {phot }}<0.2$. The most X-ray luminous AGN in our sample is irac040934, with a $L_{\text {Hard }}=10^{45} \mathrm{erg} \mathrm{s}^{-1}$ and $z_{\text {phot }}=2.42$.

The behavior of the Starburst-contaminated AGNs is very similar to that of the majority of the Type-1 AGNs and exactly 


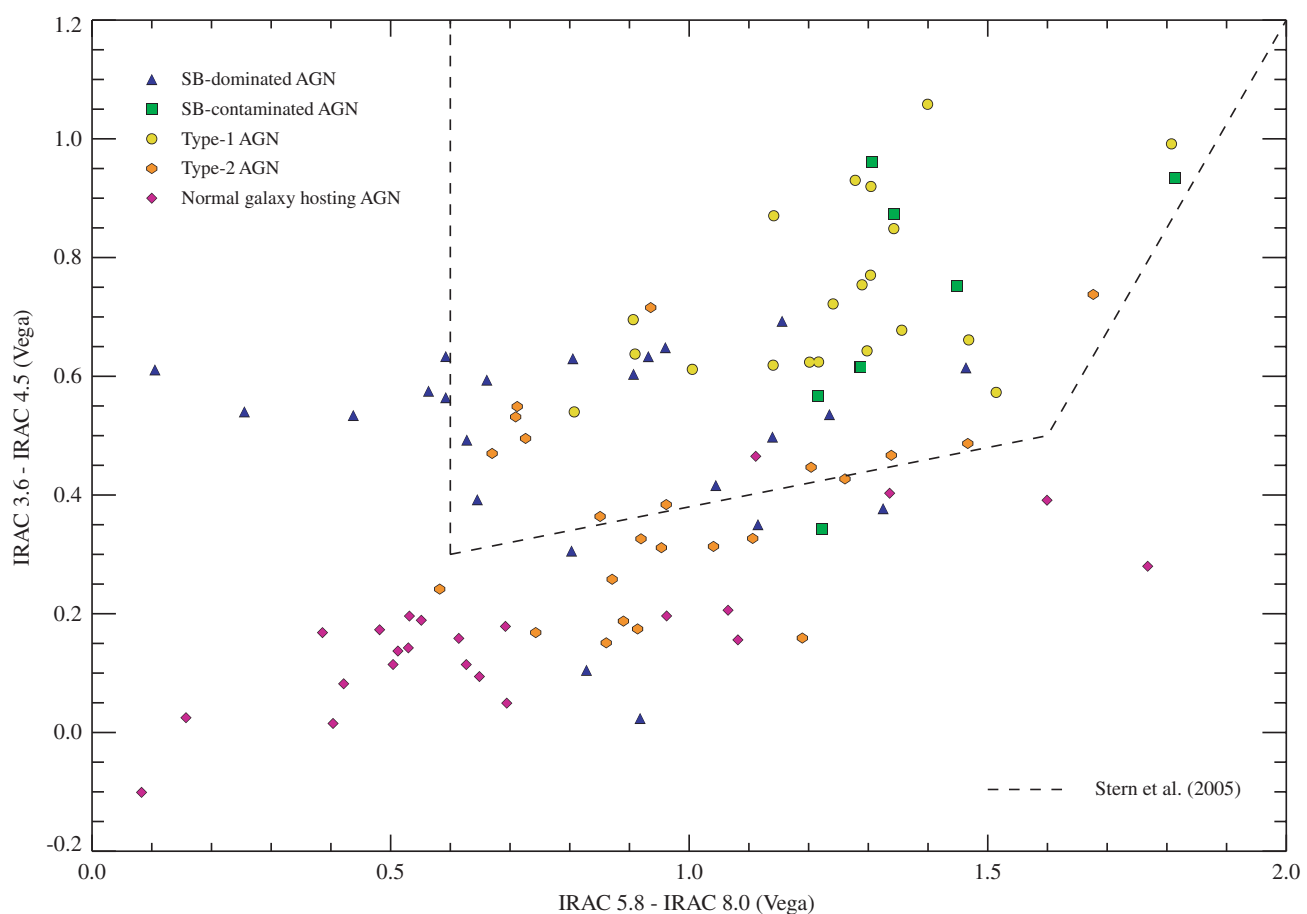

Figure 5. IRAC color-color plot for the 96 sources in our sample. Symbols are the same as in Figure 2, indicating the template fitting classification. The dashed line corresponds to the Stern et al. (2005) empirical separation of AGNs in their sample.

(A color version of this figure is available in the online journal.)

coincides with the hard X-ray luminosity range found by Alonso-Herrero et al. (2006) for BLAGN-fitted objects. This indicates that, despite the starburst appearance of the SEDs of these objects at longer wavelengths, with regard to their X-ray emission their AGN nature dominates.

The Starburst-dominated AGNs are contained in a narrower interval of X-ray luminosities ( $L_{\mathrm{Hard}}=10^{42-44} \mathrm{erg} \mathrm{s}^{-1}$ and $L_{\text {Soft }}=10^{41-44} \mathrm{erg} \mathrm{s}^{-1}$ ), although reaching high values, indicating that these galaxies are not only starbursts but also masked-AGNs that show strong X-ray emission. Indeed, very few bona fide starburst galaxies have $L_{X}>10^{42} \mathrm{erg} \mathrm{s}^{-1}$, even including luminous sources at moderate redshifts (Zezas et al. 2001). Only for warm ULIRGs luminosities of up to $10^{42} \mathrm{erg} \mathrm{s}^{-1}$ are expected (Franceschini et al. 2003). Type-2 AGNs display hard X-ray luminosities ranging from $10^{42}$ to $10^{43} \mathrm{erg} \mathrm{s}^{-1}$, staying in a much narrower range and with lower values than those of Type-1 AGNs. The values of hard X-ray luminosities that we find for Starburst-dominated AGNs and Type-2 AGNs coincide with those found by Alonso-Herrero et al. (2006) for their galaxies fitted with narrow-line AGN (NLAGN)+ULIRG templates.

Finally, the Normal galaxy hosting AGNs group shows the lowest luminosity range of any of the groups $\left(L_{\mathrm{Hard}}=L_{\mathrm{Soft}}=\right.$ $10^{40-43} \mathrm{erg} \mathrm{s}^{-1}$ ), which is consistent with the fact that they are hosting low-luminosity AGN (Dunlop et al. 2003; Kauffmann et al. 2003; Grogin et al. 2005; Pierce et al. 2007). The hard $\mathrm{X}$-ray luminosity range of this group of objects coincides with typical luminosities $\left(L_{\mathrm{Hard}}<2 \times 10^{42} \mathrm{erg} \mathrm{s}^{-1}\right)$ of the local cool ULIRGs population, except for four sources, namely, IRAC045337, IRAC019616, IRAC016716, and IRAC049420.

These results, together with the mean redshift of each group reported in Section 4.1, point out that the evolution of AGNs is luminosity-dependent, with low-luminosity AGNs peaking at lower redshifts than luminous active nuclei (Hasinger 2003; Hasinger et al. 2005; Fiore et al. 2003; Ueda et al. 2003;
LaFranca et al. 2005; Brandt \& Hasinger 2005; Bongiorno et al. 2007).

\subsection{Infrared and Optical Properties}

The IRAC mid-IR colors have been used as a diagnostic tool to separate AGNs from nonactive galaxies and stars in different samples (Lacy et al. 2004; Hatziminaoglou et al. 2005; Stern et al. 2005; Alonso-Herrero et al. 2006; Barmby et al. 2006; Donley et al. 2007). Particularly, Stern et al. (2005) show an IRAC color-color diagram for the AGES sample, with all their objects spectroscopically classified. They found that BLAGN are clearly separated from Galactic stars and ordinary galaxies in their diagram, with the NLAGN located both inside and outside the active galaxies area.

An IRAC color-color diagram for our sample is represented in Figure 5. The different symbols indicate the template fitting classification. The dashed line in Figure 5 corresponds to the Stern et al. (2005) empirical separation of AGNs in their sample. In our case, this region includes all the Type-1 AGNs, and all but one of the Starburst-contaminated AGNs. This is expected, since five of the seven galaxies belonging to that group were fitted with the Sy1/SB/ULIRG template (SED type $=4$; see Table 1), while the one located outside the AGN region was fitted with the Sy2/SB template (SED type $=6$ ).

The only galaxy classified as Starburst-contaminated AGNs fitted with a Sy2/SB/ULIRG template (SED type = 13) that is contained in the Stern et al. (2005) AGN region is irac046309, its photometric redshift being $z=2.45$. The redshift of this source is mentioned here because, as Barmby et al. (2006) discuss and illustrate in their Figure 6, the AGN-dominated templates have red mid-IR colors and thus, lie inside the Stern et al. (2005) region at all redshifts, whereas the star-forming galaxy templates begin to move into this area as the redshift increases. This explains why all Type-1 AGNs are located inside the AGN region marked by the dashed line, as well as the six Starburst- 


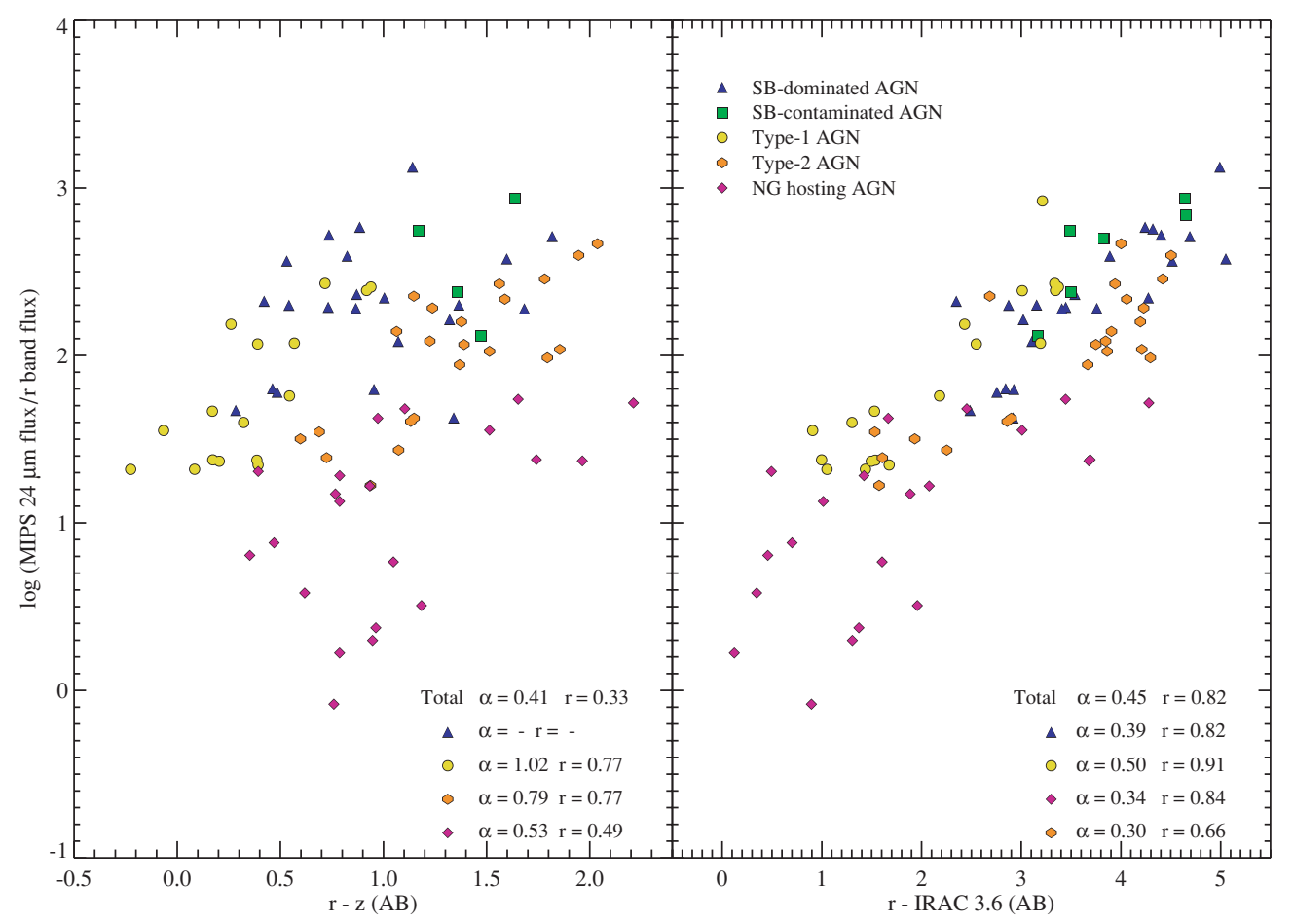

Figure 6. $\log$ (MIPS $24 \mu \mathrm{m} / r$ band) flux ratio vs. the $r-z$ (AB) color for objects with unique detection in our sample and available $r$ and $z$ magnitudes (left panel) and the same ratio vs. the $r$-IRAC $3.6 \mu \mathrm{m}$ color (right panel). The slopes and correlation coefficients are labeled for the global fit and for each of the four groups.

(A color version of this figure is available in the online journal.)

contaminated AGNs: five are fitted with the Sy1/SB/ULIRG template, and the galaxy IRAC046309 is a high redshift Sy2/ SB/ULIRG. The Normal galaxy hosting AGN (that have the bluest colors of the sample) are excluded of this region (except for one of them).

As shown in Stern et al. (2005), the active galaxy region is contaminated with Galactic stars and normal galaxies, with the NLAGN located both inside and outside this area. The same happens in our Figure 5: Starburst-dominated AGNs and Type-2 AGNs are partly contained in this area, and partly not. We have estimated the mean redshifts of both groups of galaxies for the in- and out-objects, finding that the Starburst-dominated AGNs lying outside the pure AGN region have a mean $z=$ $1.35 \pm 0.54$, while those inside have a mean $z=1.52 \pm 0.50$. Following the same trend, the Type-2 AGNs mean redshift is $z=$ $0.79 \pm 0.26$ for the outside objects, and $z=0.97 \pm 0.32$ for the galaxies included in the Stern et al. (2005) region. This is again consistent with the evolution of mid-IR colors with redshift for star-forming galaxies (Barmby et al. 2006; Donley et al. 2008). However, these mean redshifts for Starburst-dominated AGNs and Type-2 AGNs lying inside and outside the Stern et al. (2005) region are only orientative, since the differences between them are not statistically significant.

The reliability of these types of diagrams (mid-IR color selection) in selecting AGNs has been questioned in the literature (Cardamone et al. 2008; Donley et al. 2008). It seems that they fail to identify a large number of X-ray-selected AGNs, finding only the most luminous ones. In our work, the Stern et al. (2005) region wraps all the Type- 1 objects, all but one of the Starburstcontaminated AGNs, and half of the Starburst-dominated AGNs and Type-2 AGNs. Fifty-two percent of our sample is included in this area, but the low-luminosity AGNs (most of them Normal galaxy hosting AGNs and several Starburst-dominated AGNs and Type-2 AGNs) are excluded. Cardamone et al. (2008) find that $76 \%$ of their spectroscopically selected BLAGNs fall inside this region, but only $40 \%$ of the X-ray-selected objects are included. Summarizing, although the diagram in Figure 5 only includes half of our sample in the Stern et al. (2005) region, it seems very effective at segregating the different AGN groups.

The left panel of Figure 6 shows the mid-IR $24 \mu \mathrm{m}$ to optical ( $r$ band) flux ratio versus the $(r-z)_{\mathrm{AB}}$ color for galaxies with unique detection. The $24 \mu \mathrm{m}$ to optical flux ratio is a rough estimator of obscured activity in galaxies, since the $24 \mu \mathrm{m}$ sources with faint optical counterparts should be luminous AGNs obscured by dust and/or gas in the optical range (Fiore et al. 2008). The $(r-z)_{\mathrm{AB}}$ color depends on the obscuration present in the galaxy.

As expected for pure AGNs, we find a significant correlation between the $24 \mu \mathrm{m}$ to $r$ flux ratio and $(r-z)_{\mathrm{AB}}$ for the Type-1 AGNs and Type-2 AGNs, because the nuclear emission dominates both in the optical and mid-IR wavelengths (Fiore et al. 2008). However, the correlation is not significant for the Starburst-dominated AGNs group, since they have an excess in their mid-IR emission, coming from the dust heated by the starbursts, in addition to the dust heated by the AGN. Normal galaxy hosting AGNs also display a correlation between the two quantities, but with a different slope and lower correlation coefficient than the pure AGNs objects. The corresponding slopes and correlation coefficients are indicated in Figure 6, except for the Starburst-contaminated AGNs group, due to the low number of objects fitted with this set of templates.

A segregation between the different groups is noticeable in the plot: the Starburst-dominated AGN and Starburst-contaminated AGN are shifted toward the highest values of the mid-IR to optical ratio ( $\log [24 \mu \mathrm{m} / r$ band flux] greater than 1.6), Type-1 AGNs and Type-2 AGNs are located at intermediate values, and the Normal galaxy hosting AGNs have the lowest values of this ratio (log $[24 \mu \mathrm{m} / r$ band flux] $<1.8)$. Obscured AGNs should 

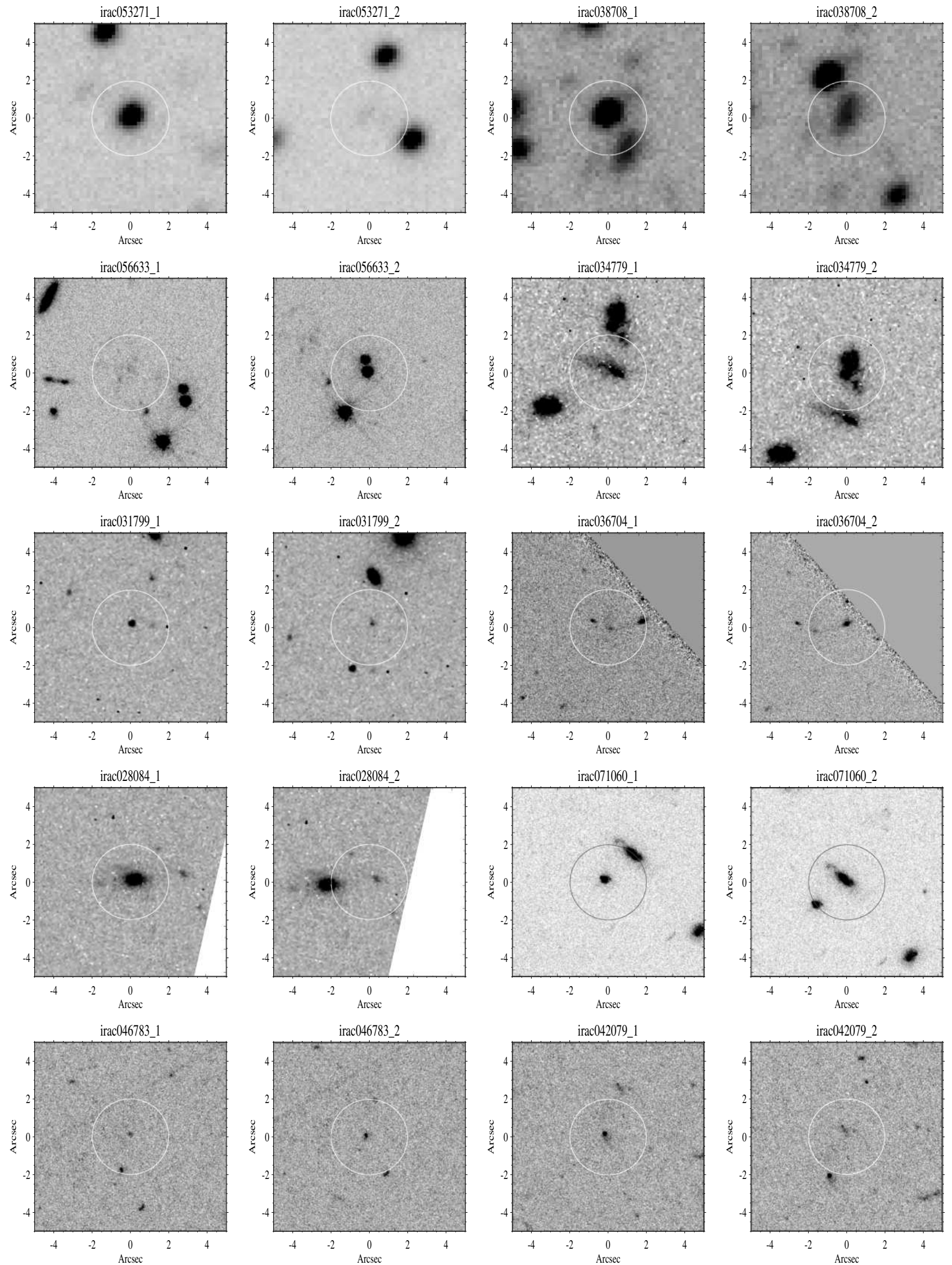

Figure 7. Optical images $\left(10^{\prime \prime} \times 10^{\prime \prime}\right)$ of the sources with double detection in our sample. For objects IRAC053271 and IRAC038708, $R$-band images from CFHTLS are shown. The rest are ACS V-band images: IRAC056633, IRAC034779, IRAC031799, IRAC036704, IRAC028084, IRAC071060, IRAC046783, and IRAC042079. Two stamps are shown for each pair of galaxies, the circle indicating the position of each source candidate. 

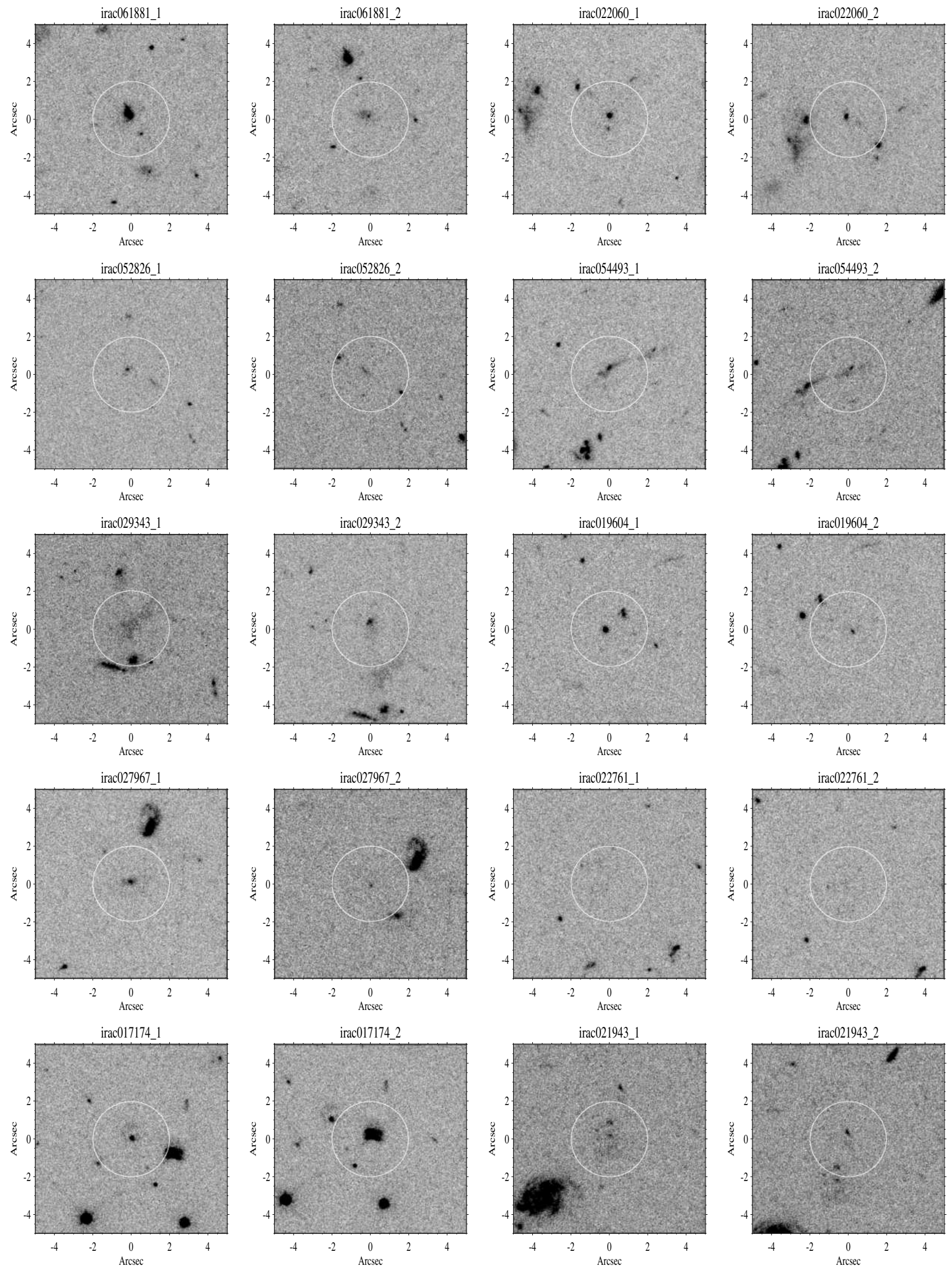

Figure 8. Same as in Figure 7 (all the images from ACS $V$ band/HST), for objects IRAC061881, IRAC022060, IRAC052826, IRAC054493, IRAC029343, IRAC019604, IRAC27967, IRAC022761, IRAC017174, and IRAC021943. 
Table 4

Morphological Classification of the 20 Objects with Double Detections. Description of the IR Contribution of Each Member in a Pair is Reported

\begin{tabular}{|c|c|c|c|c|}
\hline ID & ID IRAC & s1 IR Emission & s2 IR Emission & Comments \\
\hline 11 & 053271 & Dominant & Negligible & $\mathrm{s} 2$ diffuse region, s2 probably not $\mathrm{AGN}$ \\
\hline 13 & 038708 & Dominant & Dominant & Interacting system \\
\hline 23 & 056633 & Dominant & Dominant & s1 diffuse region with stellar knots, s2 interacting system itself \\
\hline 31 & 034779 & Dominant & Negligible & Interacting system \\
\hline 32 & 031799 & Dominant & Negligible & s1 QSO-like, s2 probably not AGN \\
\hline 34 & 036704 & Dominant & Negligible & s1 interacting system itseft, s2 probably not AGN \\
\hline 37 & 028084 & Dominant & Negligible & s1 face-on disky galaxy, s2 probably not AGN \\
\hline 39 & 071060 & Dominant & Negligible & Interacting system spectroscopically confirmed, s2 probably not AGN \\
\hline 58 & 046783 & Dominant & Dominant & s1 and s2 QSO-like \\
\hline 65 & 042079 & Dominant & Negligible & s2 probably not AGN, s2 diffuse region with stellar knots \\
\hline 88 & 061881 & Dominant & Negligible & s2 probably not AGN, s2 diffuse region with stellar knots \\
\hline 100 & 022060 & Dominant & Negligible & s1 interacting system itself, s2 probably not AGN \\
\hline 102 & 052826 & Dominant & Negligible & s2 probably not AGN, s2 diffuse region with stellar knots \\
\hline 103 & 054493 & Dominant & Negligible & Interacting system, s2 probably not AGN \\
\hline 117 & 029343 & Dominant & Negligible & s1 diffuse region and interacting system itseft, s2 probably not AGN \\
\hline 121 & 019604 & Dominant & Negligible & s1 interacting system itself, s2 probably not AGN \\
\hline 122 & 027967 & Dominant & Negligible & s2 probably not AGN \\
\hline 129 & 022761 & Dominant & Negligible & s1 and s2 optical dropouts, s2 probably not AGN \\
\hline 131 & 017174 & Dominant & Negligible & Minicluster, s2 probably not AGN \\
\hline 132 & 021943 & Dominant & Negligible & s1 diffuse regions with stellar knots, s2 probably not AGN \\
\hline
\end{tabular}

Notes. Classification of objects as "probably not AGN" is based on their mid-infrared emission.

Data presented: ID from Barmby et al. (2006), IRAC ID, mid-infrared emission of sources 1 and 2 in each pair of galaxies, and comments based on visual inspection of the objects.

be located toward the top right of Figure 6 (left panel), since they have the reddest optical colors and the highest $24 \mu \mathrm{m} / r$ band flux ratios. Starburst-dominated AGNs, Starburst-contaminated AGNs, and Type-2 AGNs fitted objects are the most obscured galaxies in our sample, according to this diagram, although they are not as obscured as those in Fiore et al. (2008). We have chosen the $(r-z)_{\mathrm{AB}}$ color instead of the most common $(r-K)_{\mathrm{AB}}$ due to the lower number of objects that have available $K$ magnitudes.

In the right panel of Figure 6 the same mid-IR $24 \mu \mathrm{m} /$ $r$ band flux ratio is shown against the $(r-\text { IRAC } 3.6 \mu \mathrm{m})_{\mathrm{AB}}$ color for galaxies with unique detection. As much as the $(r-$ $z)_{\mathrm{AB}}$ color is contaminated by the host galaxy contribution, the $(r-\text { IRAC } 3.6 \mu \mathrm{m})_{\mathrm{AB}}$ color is dominated by the hot dust emission heated by the AGN and/or intense star formation (Brusa et al. 2005). In this case, all the individual groups of objects as well as the whole sample show significant and tight correlations. The segregation between the different groups mentioned before is clear again in this graph. The Starburstdominated AGNs and Starburst-contaminated AGNs clearly show the reddest colors of the sample $(r-$ IRAC $3.6 \mu \mathrm{m}>$ 2.3), while the Normal galaxy hosting AGNs display the bluest, concentrated around $r-$ IRAC $3.6 \mu \mathrm{m} \sim 1.6$. These objects occupy the left bottom corner of the right panel of Figure 6 because the host galaxy outshines the AGN emission at all wavelengths (except in the X-rays).

\subsection{Objects with Double Detection in the Optical Bands}

Twenty out of the 116 objects that comprise our full sample show double detection in the ground-based images, thanks to their better spatial resolution. Figures 7 and 8 show Advanced Camera for Surveys (ACS) V-band/Hubble Space Telescope (HST) images of both detections (indicated with circles) for each pair of galaxies, except for objects IRAC053271 and IRAC038708, which do not have HST imaging, and for which optical CFHTLS $r$-band images are shown instead. The CFHTLS magnitudes have been employed for the calculations with the HyperZ code, although we have chosen the HST images for display purposes, because of their better resolution. These images help us classifying morphologically these 20 objects with double detections as either interacting systems, different star forming regions of the same galaxy, or simple source confusion, as described in Table 4.

In the case of these objects with double detection, for a single IRAC source, there are two counterparts in the groundbased images within the 2 .'5 search radius. As described in Section 2, the optical/NIR reference image is used to determine the positions of each source. The IRAC images are then deconvolved using the IRAC PSFs. The sources can be resolved for separations $\sim 1^{\prime \prime}$ from each other, and IRAC fluxes are then remeasured by fixing the positions of the objects in each pair, and by scaling the flux of each object in an aperture of 0.9 (Pérez-González et al. 2005, 2008a). The integrated magnitude is derived by applying an aperture correction based on empirical IRAC PSFs (for the 0.9 aperture the factors are 1.01 \pm 0.07 , $1.02 \pm 0.08,1.2 \pm 0.10$, and $1.44 \pm 0.14$ for the channels 3.6, $4.5,5.8$, and $8.0 \mu \mathrm{m}$, respectively). See the Appendix of PérezGonzález et al. (2008a) for more details. The flux contamination is found to be smaller than $10 \%$ in most cases, and even smaller for the nonIR-bright sources.

Once we know the positions of each galaxy in a pair, we can check whether the mid-IR emission comes from both, or just from one of the objects in the IRAC and MIPS images. In the majority of the cases, all the mid-IR flux in a pair of galaxies comes from only one of the objects (see Table 4), the other probably being a nonactive object. Then, we assume that the $\mathrm{X}$-ray emission comes from the same mid-IR emitter, and we calculate photometric redshifts for the active objects only.

In those cases where the mid-IR emission cannot be allocated clearly to one of the objects (IRAC038708, IRAC056633, and IRAC046783), photometric redshifts calculated by HyperZ for 
Table 5

Photometric Redshifts and Additional Information Derived from the SED Fits of the 20 Objects with Double Detections. Spectroscopic Redshifts are Reported When Available

\begin{tabular}{|c|c|c|c|c|c|c|c|c|c|c|c|}
\hline ID & ID IRAC & R.A. $\left({ }^{\circ}\right)$ & $\operatorname{Decl} .\left(^{\circ}\right)$ & $z_{\text {spec }}$ & $z_{\text {phot }}$ & $\chi_{v}^{2}$ & Prob $(\%)$ & $A_{V}$ & $L_{r}$ & Template & Group \\
\hline 11 & $053271_{-} 1$ & 214.1439 & 52.3775 & $2.089(2)$ & 2.44 & 1.29 & 23 & 1.20 & 45.21 & 14 & Type-1 AGN \\
\hline 13 & 038708_1 & 214.1499 & 52.3200 & $\ldots$ & 1.09 & 0.94 & 49 & 0.00 & 44.17 & 1 & SB-dom. AGN \\
\hline 13 & 038708_2 & 214.1499 & 52.3200 & $\ldots$ & 1.10 & 1.21 & 28 & 0.00 & 44.11 & 3 & SB-dom. AGN \\
\hline 23 & $056633_{-} 1$ & 214.2032 & 52.4330 & $\ldots$ & 1.21 & 0.35 & 95 & 0.00 & 44.30 & 5 & SB-dom. AGN \\
\hline 23 & $056633 \_2$ & 214.2032 & 52.4330 & $\ldots$ & 0.26 & 1.73 & 6 & 0.30 & 43.18 & 22 & NG hosting AGN \\
\hline 31 & 034779_1 & 214.2239 & 52.3567 & $\ldots$ & 0.22 & 0.91 & 53 & 0.90 & 42.76 & 10 & Type-2 AGN \\
\hline 34 & 036704_1 & 214.2506 & 52.3845 & $\ldots$ & 1.41 & 0.42 & 92 & 0.30 & 44.47 & 21 & NG hosting AGN \\
\hline 37 & 028084_1 & 214.2677 & 52.3611 & $\ldots$ & 0.44 & 0.89 & 55 & 0.90 & 43.48 & 10 & Type-2 AGN \\
\hline 39 & 071060_1 & 214.2738 & 52.5418 & $0.170(2)$ & 2.34 & 0.80 & 64 & 0.30 & 44.90 & 7 & Type-1 AGN \\
\hline 58 & $046783_{-} 1$ & 214.3350 & 52.4867 & $\ldots$ & 1.23 & 1.85 & 5 & 0.60 & 43.73 & 2 & SB-dom. AGN \\
\hline 58 & 046783_2 & 214.3350 & 52.4867 & $\ldots$ & 0.92 & 3.14 & 0 & 0.00 & 42.67 & 2 & SB-dom. AGN \\
\hline 65 & 042079_1 & 214.3627 & 52.4867 & $\ldots$ & 1.16 & 2.30 & 1 & 0.00 & 43.69 & 8 & Type-2 AGN \\
\hline 88 & $061881_{-} 1$ & 214.4148 & 52.6053 & $\ldots$ & 0.26 & 1.72 & 8 & 0.00 & 42.22 & 9 & Type-2 AGN \\
\hline 102 & $052826_{-} 1$ & 214.4590 & 52.6005 & $\ldots$ & 1.26 & 0.33 & 95 & 0.90 & 43.61 & 2 & SB-dom. AGN \\
\hline 103 & $054493 \_1$ & 214.4620 & 52.6093 & $\ldots$ & 2.43 & 1.79 & 6 & 0.60 & 44.43 & 12 & Type-1 AGN \\
\hline 117 & $029343_{-} 1$ & 214.4923 & 52.5262 & $\ldots$ & 1.12 & 2.09 & 2 & 0.00 & 43.71 & 2 & SB-dom. AGN \\
\hline 121 & 019604_1 & 214.5047 & 52.4950 & $0.623(1)$ & 0.14 & 1.90 & 4 & 0.00 & 41.48 & 1 & SB-dom. AGN \\
\hline 122 & $027967 \_1$ & 214.5062 & 52.5305 & $\ldots$ & 0.89 & 1.31 & 22 & 0.30 & 43.85 & 21 & NG hosting AGN \\
\hline 129 & $022761 \_1$ & 214.5373 & 52.5309 & $\ldots$ & 1.06 & 0.77 & 61 & 1.20 & 43.19 & 1 & SB-dom. AGN \\
\hline 131 & 017174_1 & 214.5545 & 52.5202 & $\ldots$ & 1.15 & 1.04 & 41 & 0.30 & 44.07 & 3 & SB-dom. AGN \\
\hline 132 & 021943 _ 1 & 214.5641 & 52.5466 & $\ldots$ & 0.98 & 0.63 & 77 & 0.30 & 43.44 & 2 & SB-dom. AGN \\
\hline
\end{tabular}

Notes. Templates are the same described in Table 1.

Data presented: ID from Barmby et al. (2006), IRAC ID, IRAC $3.6 \mu \mathrm{m}$ J2000.0 right ascension and declination, spectroscopic redshift from the DEEP public database with its corresponding reliability between brackets ( $1-2=$ low reliability, $3-4=$ high reliability), photometric redshifts for both blended galaxies when mid-infrared emission comes from both $(13,23$, and 58$)$ or for the mid-IR emitter in rest of the cases, and their corresponding $\chi_{v}^{2}$, probabilities, optical extinctions derived from the Calzetti et al. (2000) reddening law, logarithm of $\nu L_{v}$ in the $r$ band as a reference, in erg s ${ }^{-1}$, fitted templates, and general classification.

both sources in each pair have been obtained and they are reported in Table 5 together with their $\chi_{v}^{2}$, probabilities, SED type, and $A_{V}$. For the other 17 pairs of galaxies for which the mid-IR emission comes clearly from only one of the objects, we calculate photometric redshifts only for the mid-IR emitter. Spectroscopic redshifts from the DEEP database are also given when available, together with their corresponding reliability flags. Unfortunately, this is the case for only four objects, and all of them with low reliability flags ( 1 or 2 , see Table 5). Nevertheless, we can assume that the photometric redshifts, obtained as described in Section 3, are reasonably good, since we have followed the same methodology as for the 96 sources with single detections.

As reported in Table 4, IRAC056633_2, IRAC036704_1, IRAC022060_1, IRAC029343_1, and IRAC019604_1 are interacting systems themselves, as can be seen in the HST images (Figures 7 and 8). These sources must be treated with caution, since their fluxes could be contaminated with extra-emission coming from their companions. This fact explains the low probabilities of the HyperZ fits for objects IRAC056633_2, IRAC029343_1, and IRAC019604_1, reported in Table 5.

In the same way as we have done for the objects with unique detection in previous sections, we distribute here the 23 template fitted objects with double detection in the same five main categories described before. The percentages for each group are Starburst-dominated AGNs (48\% of the mid-IR emitters), Starburst-contaminated AGNs (0\%), Type-1 AGNs (17\%), Type-2 AGNs (22\%), and Normal galaxy hosting AGNs $(13 \%)$. Note that for this subsample of objects with double detection, almost half of the objects are described by starburst-type SEDs. If, as in Section 4.1., we split the objects into AGN-dominated and host-dominated galaxies, we find that $39 \%$ show AGN-like SEDs while 61\% are host-dominated, a clear over-representation. This is expected since if the pairs of galaxies are interacting objects, the number of starbursts in this subsample of galaxies should consequently increase.

\section{CONCLUSIONS}

We present a reliable method of classification of hard X-ray and mid-IR selected AGNs, based on the fit of wellsampled multiwavelength SEDs with a complete set of AGN and starburst galaxy templates. The sample studied in this paper consists of 96 AGNs with unique detection, and 20 AGNs with double detection in the EGS. The following results were found.

1. Photometric redshifts have been calculated by using the HyperZ code. The measured mean discrepancy between our $z_{\text {phot }}$ values and a subsample of highly reliable DEEP spectroscopic redshifts (flag $=3$ or 4 ) is $\Delta z=-0.03$, with $\sigma_{z}=0.11$, and 3 outliers $(8 \%)$. We provide more accurate photometric redshifts than the spectroscopic ones for objects with DEEP flag $=1$ or 2 .

2. Five main population groups have been considered according to the set of templates employed. For the 96 objects in our sample with unique detection, the following percentages have been found: Starburst-dominated AGNs (24\% of the sample), Starburst-contaminated AGNs (7\%), Type-1 AGNs (21\%), Type-2 AGNs (24\%), and Normal galaxy hosting AGNs (24\%). We find that $52 \%$ of the sample 
has AGN-dominated SEDs and the remaining $48 \%$ hostdominated SEDs.

3. Fifty-eight percent of the 96 objects with unique detection in our sample have $z_{\text {phot }}<1$, with the rest of the $z_{\text {phot }}$ of the sources distributed in a decreasing tail up to $z_{\text {phot }}=3$. The Starburst-dominated AGNs constitute the high-redshift population of the host-dominated AGNs, while the Normal galaxy hosting AGNs are concentrated at low redshifts. In the AGN-dominated group, Type-1 AGNs are randomly distributed in distance, the Starburst-contaminated AGNs are located at intermediate values of redshift, and the Type-2 AGNs are the lowest- $z$ objects.

4. An evolutionary trend is noticed, in which the Starburstdominated AGNs would be the progenitors of the Type-1 AGNs and Type-2 AGNs, via quenching of the starburst through the AGN feedback.

5. Correlations between hard/soft X-ray luminosities and UV/optical/IR data are reported for such a sample of AGNs spanning a wide range of redshift, being in this way represented the behavior of the different AGN types in the various wavelengths considered.

6. Type-1 AGNs show the highest values of hard and soft X-ray luminosities of the sample, together with the Starburst-contaminated AGNs, whilst the Normal galaxy hosting AGNs majority are concentrated at the lowest values, coinciding with the local cool ULIRGs' typical hard $\mathrm{X}$-ray luminosities. Starburst-dominated AGNs and Type2 AGNs present intermediate values of $\mathrm{X}$-ray emission, very similar to those of warm ULIRGs. This is consistent with a luminosity-dependent evolution of AGNs, with lowluminosity AGNs peaking at lower redshifts than luminous active nuclei.

7. Type-1 AGNs are all contained in the IRAC color-color diagram region empirically determined by Stern et al. (2005) for spectroscopically selected AGNs. There are many Type-2 AGNs and Starburst-dominated AGNs inside this AGN region. These objects have higher mean redshifts than those in the same group but outside the pure-AGN area, according with the evolution of the mid-IR colors with redshift for star-forming galaxies described in Barmby et al. (2006).

8. Mid-IR $24 \mu \mathrm{m}$ to optical $r$ band flux ratio versus the $(r-$ $z)_{\mathrm{AB}}$ or the $(r-\operatorname{IRAC} 3.6 \mu \mathrm{m})_{\mathrm{AB}}$ colors show a clear segregation of the different groups in both diagrams. Starburstdominated AGNs and Starburst-contaminated AGNs are displaced toward the highest values of the mid-IR to optical ratio and display the reddest colors. Type-1 AGNs and Type-2 AGNs are located at intermediate values, and the Normal galaxy hosting AGNs have the lowest values of the $24 \mu \mathrm{m} / r$ flux ratio and the bluest colors.

9. A tentative classification of objects with double detection into the five main population groups considered through this paper shows an increase of the Starburst-dominated AGNs of up to $48 \%$, while the others decrease. Sixty-one percent of the fitted objects show AGN-like SEDs, while $39 \%$ is host-dominated.

NASA's Chandra X-Ray Observatory was launched in July 1999. The Chandra Data Archive (CDA) is part of the Chandra $\mathrm{X}$-Ray Center (CXC) which is operated for NASA by the Smithsonian Astrophysical Observatory.

This work is based on observations obtained with $X M M$ Newton, an ESA Science Mission with instruments and contributions directly funded by ESA Member States and NASA.

GALEX (Galaxy Evolution Explorer) is a NASA Small Explorer, launched in 2003 April. We gratefully acknowledge NASA's support for construction, operation, and science analysis of the GALEX Mission, developed in cooperation with the Centre National d'Etudes Spatiales of France and the Korean Ministry of Science and Technology.

This work is based in part on observations made with the Spitzer Space Telescope, which is operated by the Jet Propulsion Laboratory, California, Institute of Technology under a contract with NASA.

This work is based on observations obtained with MegaPrime/ MegaCam, a joint project of CFHT and CEA/DAPNIA, at the Canada-France-Hawaii Telescope (CFHT) which is operated by the National Research Council (NRC) of Canada, the Institut National des Science de l'Univers of the Centre National de la Reserche Scientifique (CNRS) of France, and the University of Hawaii. This work is based in part on data products produced at TERAPIX and the Canadian Astronomy data Centre as part of the CFHT Legacy Survey, a collaborative project of NRC and CNRS.

This work is based on observations obtained at the Hale Telescope, Palomar Observatory, as part of a collaborative agreement between the California Institute of Technology, its divisions Caltech Optical Observatories and the Jet Propulsion Laboratory (operated for NASA), and Cornell University.

Many images of this article are based on observations made with the NASA/ESA Hubble Space Telescope, obtained from the data archive at the Space Telescope Science Institute (STScI). STScI is operated by the Association of Universities for Research in Astronomy, Inc., under NASA contract NAS526555.

This work uses data obtained with support of the National Science Foundation grants AST 95-29028 and AST 00-71198.

This work is partially funded by PN AYA2007-67965-C0301, PN AYA2006-02358, and by the Spanish MEC under the Consolider-Ingenio 2010 Program grant CSD2006-00070: First Science with the GTC (http://www.iac.es/consolider-ingeniogtc/). P.G.P.-G. acknowledges support from the Ramón y Cajal Program financed by the Spanish Government and the European Union. C.R.A., J.R.E., G.B., J.G., and P.G.P.-G. acknowledge Roser Pelló, Antonio Cabrera Lavers, and Casiana Muñoz Tuñon for their valuable help. We finally appreciate the very useful report of the anonymous referee.

\section{REFERENCES}

Alexander, D. M., et al. 2003, AJ, 125, 383

Alonso-Herrero, A., et al. 2004, ApJS, 154, 155

Alonso-Herrero, A., et al. 2006, ApJ, 640, 167

Babbedge, T. S. R., et al. 2004, MNRAS, 353, 654

Barger, A. J., Cowie, L. L., Mushotzky, R. F., \& Richards, E. A. 2001, AJ, 121, 662

Barger, A. J., Cowie, L. L., Mushotzky, R. F., Yang, Y., Wang, W.-H., Steffen, A. T., \& Capak, P. 2005, AJ, 129, 578

Barmby, P., et al. 2006, ApJ, 642, 126

Bolzonella, M., Miralles, J.-M., \& Pelló, R. 2000, A\&A, 363, 476

Bongiorno, A., et al. 2007, A\&A, 472, 443

Boulade, O., et al. 2003, Proc. SPIE, 4841, 72

Brandt, W. N., \& Hasinger, G. 2005, ARA\&A, 43, 827

Brusa, M., et al. 2005, A\&A, 432, 69

Bundy, K., et al. 2006, ApJ, 651, 120

Bundy, K., et al. 2008, ApJ, 681, 931

Calzetti, D., et al. 2000, ApJ, 533, 682

Cardamone, C. N., et al. 2008, ApJ, 680, 130 
Davis, M., et al. 2007, ApJ, 660, 1

Dickinson, M., et al. 2001, BAAS, 33, 820

Donley, J. L., Rieke, G. H., Pérez-González, P. G., \& Barro, G. 2008, ApJ, 687, 111

Donley, J. L., Rieke, G. H., Pérez-González, P. G., Rigby, J. R., \& AlonsoHerrero, A. 2007, ApJ, 660, 167

Donley, J. L., Rieke, G. H., Rigby, J. R., \& Pérez-González, P. G. 2005, ApJ, 634, 169

Dunlop, J. S., McLure, R. J., Kukula, M. J., Baum, S. A., O’Dea, C. P., \& Hughes, D. H. 2003, MNRAS, 340, 1095

Dye, S. 2008, MNRAS, 389, 1293

Eisenhardt, P., et al. 2004, ApJS, 154, 48

Fiore, F., et al. 2003, A\&A, 409, 79

Fiore, F., et al. 2008, ApJ, 672, 94

Franceschini, A., et al. 2003, MNRAS, 343, 1181

Franceschini, A., et al. 2005, AJ, 129, 2074

George, I. M., Turner, T. J., Yaqoob, T., Netzer, H., Laor, A., Mushotzky, R. F., Nandra, K., \& Takahashi, T. 2000, ApJ, 531, 52

Granato, G. L., De Zotti, G., Silva, L., Bressan, A., \& Danese, L. 2004, ApJ, 600,580

Grogin, N. A., et al. 2005, ApJ, 627, 97

Groth, E. J., et al. 1994, BAAS, 26, 1403

Hasinger, G. 2003, in AIP Conf. Proc. 666, The Emergence of Cosmic Structure, ed. S. S. Holt \& C. S. Reynolds (Melville, New York: AIP), 227

Hasinger, G. 2004, Proc. Nucl. Phys. Suppl., 132, 86

Hasinger, G., Miyaji, T., \& Schmidt, M. 2005, A\&A, 441, 417

Hatziminaoglou, E., et al. 2005, AJ, 129, 1198

Hornschemeier, A. E., et al. 2001, ApJ, 554, 742

Jannuzi, B. T., \& Dey, A. 1999, in ASP Conf. Ser. 191, Photometric Redshifts and the Detection of High Redshift Galaxies, ed. R. Weymann et al. (San Francisco, CA: ASP), 11

Kauffmann, G., et al. 2003, MNRAS, 346, 1055

Kitsionas, S., Hatziminaoglou, E., Georgakakis, A., \& Georgantopoulos, I. 2005, A\&A, 434, 475

Kron, R. G. 1980, ApJS, 43, 305

Krumpe, M., et al. 2007, A\&A, 466, 41

Kuraszkiewicz, J. K., et al. 2003, ApJ, 590, 128

Lacy, M., Petric, A. O., Sajina, A., Canalizo, G., Storrie-Lombardi, L. J., Armus, L., Fadda, D., \& Marleau, F. R. 2007, AJ, 133, 186

Lacy, M., et al. 2004, ApJS, 154, 166

LaFranca, F., Franceschini, A., Cristiani, S., \& Vio, R. 1995, A\&A, 299, 19
LaFranca, F., et al. 2005, ApJ, 635, 864

Lonsdale, C. J., et al. 2003, PASP, 115, 897

Mainieri, V., et al. 2002, A\&A, 393, 425

Martínez-Sansigre, A., Rawlings, S., Lacy, M., Fadda, D., Marleau, F. R., Simpson, C., Willott, C. J., \& Jarvis, M. J. 2005, Nature, 436, 666

Martínez-Sansigre, A., et al. 2007, MNRAS, 379, L6

Mushotzky, R. 2004, in Suppermassive Black Holes in the Distant Universe, ed. A. J. Barger (Dordrecht: Kluwer), 53

Nandra, K., et al. 2005, MNRAS, 356, 568

Park, S. Q., et al. 2008, ApJ, 678, 744

Pérez-González, P. G., Trujillo, I., Barro, G., Gallego, J., Zamorano, J., \& Conselice, C. J. 2008a, ApJ, 687, 50

Pérez-González, P. G., et al. 2005, ApJ, 630, 82

Pérez-González, P. G., et al. 2008b, ApJ, 675, 234

Peterson, K. C., Gallagher, S. C., Hornschemeier, A. E., Muno, M. P., \& Bullard, E. C. 2006, AJ, 131, 133

Piccinotti, G., Mushotzky, R. F., Boldt, E. A., Holt, S. S., Marshall, F. E., Serlemitsos, P. J., \& Shafer, R. A. 1982, ApJ, 253, 485

Pierce, C. M., et al. 2007, ApJ, 660, 19

Polletta, M., et al. 2007, ApJ, 663, 81

Risaliti, G., Maiolino, R., \& Salvati, M. 1999, ApJ, 522, 157

Schawinski, K., et al. 2007, MNRAS, 382, 1415

Springel, V., Di Matteo, T., \& Hernquist, L. 2005, MNRAS, 361, 776

Steffen, A. T., et al. 2006, AJ, 131, 2826

Steidel, C. C., Adelberger, K. L., Giavalisco, M., Dickinson, M., \& Pettini, M. 1999, ApJ, 519, 1

Stern, D., et al. 2005, ApJ, 631, 163

Strateva, I. V., Brandt, W. N., Schneider, D. P., Vanden Berk, D. G., \& Vignali, C. 2005, AJ, 130, 387

Szokoly, G. P., et al. 2004, ApJS, 155, 271

Ueda, Y., Akiyama, M., Ohta, K., \& Miyaji, T. 2003, ApJ, 598, 886

Vignali, C., Brandt, W. N., \& Schneider, D. P. 2003, AJ, 125, 433

Villar, V., et al. 2008, ApJ, 677, 169

Waddington, I., Dunlop, J. S., Peacock, J. A., \& Windhorst, R. A. 2001, MNRAS, 328,882

Waskett, T. J., et al. 2003, MNRAS, 341, 1217

Weiner, B. J., et al. 2005, ApJ, 620, 595

Wilkes, B. J., Tananbaum, H., Worrall, D. M., Avni, Y., Oey, M. S., \& Flanagan, J. 1994, ApJS, 92, 53

Zezas, A., Alonso-Herrero, A., \& Ward, M. J. 2001, Ap\&SS, 276, 601

Zheng, W., et al. 2004, ApJS, 155, 73 